University of Wollongong

Research Online

Australian Institute for Innovative Materials -

Papers

Australian Institute for Innovative Materials

$1-1-2018$

Hydration Layer Structure of Biofouling-Resistant Nanoparticles

Paul J. Molino

University of Wollongong, pmolino@uow.edu.au

Dan Yang

University of Wollongong, dy996@uowmail.edu.au

Matthew Penna

RMIT Melbourne, matthew.penna@rmit.edu.au

Keisuke Miyazawa

Kanazawa University

Brianna Knowles

University of Wollongong, bs921@uowmail.edu.au

See next page for additional authors

Follow this and additional works at: https://ro.uow.edu.au/aiimpapers

Part of the Engineering Commons, and the Physical Sciences and Mathematics Commons

Research Online is the open access institutional repository for the University of Wollongong. For further information contact the UOW Library: research-pubs@uow.edu.au 


\title{
Hydration Layer Structure of Biofouling-Resistant Nanoparticles
}

\author{
Abstract \\ Hydrophilic surface chemistries can strongly bind water to produce surfaces that are highly resistant to \\ protein adsorption and fouling. The interfacial bound water and its distinct properties have intrigued \\ researchers for decades, yet the relationship between the water three-dimensional structure and function \\ in antifouling coatings remains elusive. Here, we use hydrophilic, epoxy organosilane modified silica \\ nanoparticles to demonstrate cheap, robust, and practically applied coatings that we discover have broad- \\ ranging, ultralow fouling properties when challenged by various proteins, bacteria, and fungal spores. To \\ understand their excellent antifouling properties, frequency modulation-atomic force microscopy is used \\ to directly observe the interfacial water structure at subatomic resolution, which we validate using all- \\ atom molecular dynamic simulations that strikingly predict similar structures of water layers on the \\ original and ultralow fouling surfaces. The convergence of experimental and modeling data reveals that \\ suitably spaced, flexible chains with hydrophilic groups interact with water molecules to produce a \\ connective, quasi-stable layer, consisting of dynamic interfacial water, that provides a basis for antifouling \\ performance of ultrathin, hydrophilic surface chemistries. \\ Disciplines \\ Engineering | Physical Sciences and Mathematics \\ Publication Details \\ Molino, P. J., Yang, D., Penna, M., Miyazawa, K., Knowles, B. R., MacLaughlin, S., Fukuma, T., Yarovsky, I. \& \\ Higgins, M. J. (2018). Hydration Layer Structure of Biofouling-Resistant Nanoparticles. ACS Nano, 12 (11), \\ 11610-11624.
}

\section{Authors}

Paul J. Molino, Dan Yang, Matthew Penna, Keisuke Miyazawa, Brianna Knowles, Shane A. MacLaughlin, Takeshi Fukuma, Irene Yarovsky, and Michael J. Higgins 


\section{Hydration Layer Structure of Biofouling Resistant}

\section{Nanoparticles}

*Paul J. Molino ${ }^{1,2},{ }^{*}$ Dan Yang ${ }^{1,2}$, Matthew Penna ${ }^{3}$, Keisuke Miyazawa ${ }^{4}$,Brianna R. Knowles ${ }^{1,5}$, Shane MacLaughlin ${ }^{1,5}$, Takeshi Fukuma ${ }^{4}$, Irene Yarovsky ${ }^{1,3} \psi$ and Michael J. Higgins $^{1,2 \psi}$

1. ARC Industrial Transformation Research Hub for Australian Steel Manufacturing, NSW 2522, Australia

2. ARC Centre for Electromaterials Science (ACES), Intelligent Polymer Research Institute/AlIM Faculty, Innovation Campus, Squires Way, University of Wollongong, NSW 2522, Australia

3. School of Engineering, RMIT University, Melbourne, Victoria 3001, Australia

4. Division of Electronic Eng. and Computer Science, Kanazawa University, Kakumamachi, Kanazawa 920-1192, Japan

5. BlueScope Innovation Laboratories, Old Port Road, Port Kembla, NSW 2505, Australia.

*Joint first author

$\psi$ Corresponding Authors:

Prof. Michael Higgins

ARC Centre of Excellence for Electromaterials Science Intelligent Polymer Research Institute/AIIM Faculty Innovation Campus, Squires Way

University of Wollongong NSW 2522

T +61 242213989

Emhiggins@uow.edu.au

W electromaterials.edu.au

Prof. Irene Yarovsky

School of Engineering, RMIT University, Melbourne, Victoria 3001, Australia T +61 399252571

E: irene.yarovsky@rmit.edu.au 


\section{Abstract}

Hydrophilic surface chemistries can strongly bind water to produce surfaces that are highly resistant to protein adsorption and fouling. The interfacial bound water and its unique properties have intrigued researchers for decades yet the relationship between the water three-dimensional structure and function in antifouling coatings remains elusive. Here, we use hydrophilic, epoxy organosilane modified silica nanoparticles to demonstrate cheap, robust and practically applied coatings that we discover have broad-ranging, ultra-low fouling properties when challenged by various proteins, bacteria and fungal spores. To understand their remarkable antifouling properties, frequency modulation-atomic force microscopy is used to directly observe the interfacial water structure at sub-atomic resolution, which we validate using all-atom molecular dynamic simulations that strikingly predict similar structures of water layers on the original and ultra-low fouling surfaces. Unprecedented convergence of experimental and modelling data reveal that suitably spaced, flexible chains with hydrophilic groups interact with water molecules to produce a connective, quasi-stable layer, consisting of dynamic interfacial water, that provides a basis for antifouling performance of ultrathin, hydrophilic surface chemistries.

Keywords: antifouling, interfacial water, hydration forces, glycidoxypropyltrimethoxysilane (GPS), silica nanoparticles (SiNP), frequency modulationatomic force microscopy (FM-AFM), molecular dynamic (MD) simulations 


\section{Main Text}

Biological fouling of artificial surfaces occurs through the adhesion and colonisation by microbial and macrofouling organisms. These processes are an enormous problem for many diverse industries, including potable water treatment and transport, biomedical devices and hospital surfaces, food production, aquaculture, and maritime shipping, resulting in substantial social and economic impacts. For example, hospital acquired infections caused by the spread of pathogenic bacteria account for over 90,000 deaths and estimated to cost $\$ 35-45$ billion per year in the U.S alone ${ }^{1-2}$, while microbial slime fouling layers produced by marine bacteria and microalgae on ship hulls can increase fuel costs by up to $10 \%{ }^{3}$, equating to fuel penalties of billions of dollars globally every year in addition to increased toxic $\mathrm{NO}_{\mathrm{x}}$ and $\mathrm{SO}_{\mathrm{x}}$ emissions from low quality diesel fuels ${ }^{4}$.

Existing antifouling technologies have relied on the incorporation of toxic biocidal compounds, including heavy metals, to kill target fouling organisms. However, legislative changes in recent years have signalled the gradual outlawing of many such compounds ${ }^{5}$, including tributyltin. There are also ongoing concerns over the potential development of biocide resistant pathogenic organisms from some widely used heavy metals, including copper and silver. The need for greener technologies has seen an increased focus on the development of environmentally benign coatings that function via tailoring of material surface energy and/or topography to offer self-cleaning or fouling resistant properties ${ }^{6}$. Of particularly critical importance is the identification and development of antifouling materials and chemistries that are economically viable, scalable, and can be easily incorporated into existing or next-generation fabrication processes. 
Hydrophilic polymers designed to strongly bind water have been extensively studied as an effective surface layer or coating to prevent biofouling through the formation of a steric and energetic barrier to biomolecular adsorption ${ }^{7}$. These systems include poly(ethylene glycol) (PEG) that engages in hydrogen bonding with water via the ether oxygen, and zwitterion based chemistries that bind water more strongly via an ionic solvation mechanism. Experimental approaches have confirmed the presence of hydration layers associated with hydrophilic polymer-based surfaces ${ }^{7}$ yet the fundamental mechanisms underlying their capacity to inhibit surface fouling and how such hydration layers differ from equally hydrophilic interfaces that do not prevent surface fouling is still unclear. Furthermore, there have been no detailed experimental studies that have adequately elucidated the 2- and 3-dimensional structural organisation and properties of hydration layers on low fouling hydrophilic surfaces. In the absence of detailed molecularlevel experimental data, all-atom classical molecular dynamic (MD) simulations have been employed to provide an insight into water structure on surfaces with experimentally unachievable atomistic resolution ${ }^{8}$. To bridge this length scale gap, novel advances in experimental tools are required to converge with all atom modelling and resolve the 2D and 3D network water structure and dynamics with molecular resolution.

This study aims to resolve molecular water structure on chemically modified commercially available silica nanoparticles (SiNP) functionalized with glycidoxypropyltrimethoxysilane (GPS), an epoxy organosilane commonly used as a coupling agent or binder in colloidal silica coatings ${ }^{9}$ and, as demonstrated herein, discovered to be biofouling resistant. The GPS functionality is a short-chain with internal ether oxygen and end epoxide group that can be opened via hydrolysis to form a vicinal diol ${ }^{10-11}$, presenting a 
hydrophilic surface chemistry (Figure 1b). The GPS modified SiNPs are used to demonstrate cheap, robust and practically applied coatings that we prove to have broad-ranging, ultralow fouling properties when challenged by various proteins, bacteria and fungal spores. Specifically, we challenged the GPS modified SiNP surfaces against the pathogenic bacterium Escherichia coli which is known to cause disease and infection, and spores of the fungi Epicoccum nigrum, a known biodeteriogen that affects terrestrial man-made materials and surfaces. To understand their remarkable antifouling properties, we apply 3D Frequency Modulation-Atomic Force Microscopy (3D FM-AFM) ${ }^{12-13}$ to directly resolve the 3D interfacial water structure encapsulating the surface of single nanoparticles, and to measure the forces associated with these structures and experienced in the final $\mathrm{nm}$ 's above the nanoparticle surface. With the experimental data achieving structural resolution on the order of subangstroms, we validate the observations on comparable length scales using all-atom MD simulations, which strikingly predict similar structuring of water layers on the original and ultra-low fouling surfaces.

\section{Results and Discussion}

Surface coatings were fabricated using a commercially available $\mathrm{SiO}_{2}$ nanoparticle (SiNP) dispersion, Bindzil ${ }^{\oplus}$, a $7 \mathrm{~nm}$ silica sol with $\left(\operatorname{cc301^{\oplus }} \& \operatorname{cc} 302^{\circ}\right)$ or without $\left(30 / 360^{\circ}\right)$ surface functionalisation with glycidoxypropyltrimethoxysilane (GPS). Thermogravimetric analysis (TGA) confirmed the density of GPS functionalisation on functionalised particles, referred to as low-density and high-density for Bindzil $c c 301^{\circ}$ and $c c 302^{\circ}$, respectively (Supplemental

Figure S1). SiNP coatings were created by first dispersing SiNPs in deionised water (4wt\%), followed by using spin coating to fabricate a thin SiNP coating. Scanning electron microscopy (SEM) images show homogenous surface coatings were produced on model gold 
coated substrates, with thickness of $\sim 100 \mathrm{~nm}$ and low surface roughness of $<\sim 5 \mathrm{~nm}$ r.m.s (data no shown) (Figure 1a and Supplemental Figure S1). All SiNP surfaces presented a hydrophilic interface, with static water contact angles below $22^{\circ}$ (Supplemental Figure S1).

\section{Protein and Microbial Cell Adhesion to SiNP Coatings}

Quartz crystal microbalance with dissipation monitoring (QCM-D) was used to examine the adsorption of three model proteins to the various SiNP coatings deposited on QCM-D sensors (Figure 1c-e). Bovine serum albumin (BSA) is a stable yet conformationally dynamic small plasma protein, fibrinogen $(\mathrm{Fb})$ is an important protein in biomedicine implicated in clot formation and wound healing, and hydrophobin $(\mathrm{Hb})$ is a cell surface protein used by fungal spores to adhere and grow on surfaces. BSA adsorption was significantly reduced by $94 \%\left(44 \pm 19 \mathrm{ng} \cdot \mathrm{cm}^{-2}\right)$ for the low-density $\operatorname{cc} 301^{\circledR}$ coatings and $96 \%$ $\left(27 \pm 4 \mathrm{ng} . \mathrm{cm}^{-2}\right)$ for the high-density $\operatorname{cc} 302^{\circledR}$ coatings relative to coatings composed of the unmodified $30 / 360^{\circ}$ SiNPs $\left(806 \pm 36 \mathrm{ng} \cdot \mathrm{cm}^{-2}\right.$ ) (Figure 1c). Fb adsorption was also dramatically reduced on coatings of the GPS functionalised SiNP, demonstrating a reduction in protein binding of $92 \%\left(132 \pm 107 \mathrm{ng} . \mathrm{cm}^{-2}\right)$ and $97 \%\left(39 \pm 12 \mathrm{ng} . \mathrm{cm}^{-2}\right)$ for low-density $\operatorname{cc} 301^{\circ}$ and high-density $\operatorname{cc} 302^{\circ}$ SiNP surfaces, respectively, compared to the $30 / 360^{\circ}$ unmodified SiNP coating (1719 $\left.\pm 225 \mathrm{ng} . \mathrm{cm}^{-2}\right)$ (Figure 1d). Hb adsorption was reduced to undetectable levels on the low-density $\operatorname{cc} 301^{\circ}$ and high-density $\operatorname{cc} 302^{\circ}$ surfaces compared to the unmodified SiNP $\left(280 \pm 100 \mathrm{ng} \cdot \mathrm{cm}^{-2}\right)$ measured using the QCM-D technique (Figure 1e), thus demonstrating ultra-low fouling properties, i.e. $<5 \mathrm{ng} . \mathrm{cm}^{-2}$, against this protein.

Microbial adhesion of two problematic microbial fouling organisms from different kingdoms were significantly impeded on the GPS modified SiNP surfaces. Adhesion of the bacteria Escherichia coli was reduced by $98.9 \%$ and $99.7 \%$ on low-density $\operatorname{cc} 301^{\circledR}$ and high- 
density $\operatorname{cc} 302^{\circledR}$ relative to the $30 / 360^{\circledR}$ unmodified SiNP substrates, respectively (Figure 1fh). Adhesion of spores of the fungi Epicoccum nigrum was significantly reduced on the GPS modified SiNP coatings, with the percentage of spore coverage on the low-density $\operatorname{cc} 301^{\circ}$ $(0.51 \%)$ and high-density $\operatorname{cc} 302^{\circ}(0.11 \%)$ coatings significantly less than that on the $30 / 360^{\circ}$ unmodified SiNP coating (1.6\%) (Figure 1i-k).

\section{D FM-AFM characterisation of hydration layers on SiNP surfaces}

To gain further insight into the molecular mechanism of the broad-ranging, antifouling properties of the GPS-modified SiNPs, 3D FM-AFM was used to characterize the interfacial structure and properties above single nanoparticles of the coatings. FM-AFM operates by oscillating a cantilever tip with an amplitude on the order of nanometers, sensing atomic and molecular forces as it moves in the z-dimension very close to the substrate surface ${ }^{14}$ and previously shown to detect single water layers on various surfaces, including crystal substrates, e.g. mica, polydiacetylene ${ }^{15}$, lipid bilayers ${ }^{16-18}$ and protein layers ${ }^{19}$. Recently developed by Fukuma et al. ${ }^{12-13,20-21}$, 3D FM-AFM uses oscillation amplitudes of 1-2 $\AA$ to build up a $2 D$ array of these measurements that generates 3D images of the spatial distribution of ions and structured water networks, including their forces and energies, within approximately $2 \mathrm{~nm}$ from the surface. In our experiments, the cantilever was firstly precisely located above a single nanoparticle of a coating and the 3D FM-AFM measurements configured to perform 3D volume maps with resolution of $4 \mathrm{~nm} \times 4 \mathrm{~nm} \times 2$ $\mathrm{nm}$ (length, width, height) in a $10 \mathrm{mM} \mathrm{NaCl}$ solution. 
The 3D volume maps show distinct, continuous layer structures occurring in the immediate vicinity of the surface and across the entire $X Y$ plane of both the unmodified and high-density $\operatorname{cc} 302^{\circ}$ GPS modified SiNP surfaces (Supplemental Figure S2). The 3D volume maps can be further separated into 2D cross-sections, 2D top-view images and 1D profiles to more closely examine the structure and properties of the interfacial layer phase. By taking vertical slices through the $\mathrm{Z}$ plane in the $\mathrm{X}$ or $\mathrm{Y}$ direction, $2 \mathrm{D}$ cross-sections reveal the interfacial layer phase consists of discrete, molecular structures extending to $\approx 5.0 \AA$ from the surface of the $30 / 360^{\circ}$ unmodified SiNP (Figure $2 \mathrm{a}(\mathbf{i})$ ). At a maximum of $2 \mathrm{~nm}$ above the surface down to the structured interfacial layers, no response in the frequency signal is indicative of the bulk water phase (Figure $\mathbf{2}$ a (i-iii)). As the cantilever approaches further to toward the SiNP surfaces, changes in the magnitude of the frequency signal and its XYZ spatial dependence denotes the interfacial layer phase. These structures originate from both an increase and decrease in the frequency signal, relative to the bulk water phase $(\Delta f=$ $0 \mathrm{~Hz}$ ), to form layers of high frequency contrast with interposing darker regions (Figure 2a). The layers are not always strictly horizontal to the surface and in some regions propagate at different angles from the surface (Figure $\mathbf{2} \mathbf{f} \mathbf{i}$ and $\mathbf{i i}$ ) to give a 'zig-zag' appearance and more generally an undulating profile with molecular-scale height variations. Serial 2D crosssections in either the $\mathrm{X}$ or $\mathrm{Y}$ direction show that this interfacial structured water layer occurs throughout the 3D volume map (Figures $\mathbf{2}, \mathbf{b}$-e), therefore producing an intricate molecular architecture across the entirety of a $30 / 360^{\circ}$ unmodified SiNP surface. Lower lateral resolution $2 \mathrm{D}$ cross-sections with height correction can also be visualized, enabling clearer superimposition and reference of the interfacial layer structures to the curvature and/or roughness of the nanoparticles within the coating (Figure $\mathbf{2 f}$ ). 
1D profiles plotting the frequency shift versus z-distance taken at different XY positions reveal an oscillatory profile that is commonly associated with the force required by the AFM tip to sequentially displace individual, structured water layers close a surface ${ }^{22}$ (Figure 2ae). Specifically, 1-2 oscillations occurring within $<\approx 5.0 \AA$ from the surface, with peak widths equating to the dimension of a single water molecule, i.e. $2.0-2.5 \AA$, confirms the presence of water layers responsible for the interfacial layer structure on the unmodified SiNP (Figure 2a (ii,iii)). The width of an oscillatory peak delineates the single water layer thickness in the $2 \mathrm{D}$ cross-sections, while the magnitude of their frequency, or effectively a force of $\sim 100 \mathrm{pN}$ per oscillation, can be integrated with respect to $z$-distance to give an energy cost of $\sim 0.1$ $0.2 \mathrm{eV}$ for displacing a single water layer (Supplementary Figure S3). To date, reasons for variations in the number of oscillatory peaks and their differing z-distances from the surface have been unclear in AFM-based force measurements though may now be explained by 3D FM-AFM due to the ability to correlate 1D profiles with the force-constructed atomic and molecular structures revealed in $2 \mathrm{D}$ cross-sections ${ }^{15}$. It is clear that the presence of water layer(s) bearing intricate, lateral structural variations at the molecular-level produces such variations in oscillatory profiles, which is further evident in a comparison of different 1D profiles (Figure $\mathbf{2 b - e )}$.

The 2D trajectory of the water layers across the unmodified SiNP can be visualized by taking a horizontal slice through the 3D volume maps to provide top-view images at a specified z-distance (Figure 3). Evolution of the serial slices is viewed when moving from the SiNP surface ( $z$-distance $=0 \AA$ ) through to the interfacial structured water layers ( $z$-distance = $2.5 \AA$ ) and then into bulk water up to the maximum z-distance of $0.7 \mathrm{~nm}$ (Figure 3). 
Specifically, at a z-distance $=2.5 \AA$, the interfacial water layer shows a continuous, branched or network-like water structure, indicated by brighter frequency contrast, with the interposing darker contrast regions (Figure 3(ii)). This complex, network-like water structure is very distinct from that of highly-ordered structures on solid, flat, crystalline surfaces, such as mica, fluorite, and calcite, that template the lateral arrangement of individual water molecules according to their hexagonal atomic lattices ${ }^{23-24}$. 3D FM-AFM experiments show local hydration regions corresponding to specific atomic sites such as $\mathrm{Ca}^{2+}$ and $\mathrm{F}^{-}$of fluorite (111) surfaces, and $\mathrm{Ca}^{2+}$ and $\mathrm{CO}^{2-}{ }_{3}$ of calcite (1014) surfaces, as well as interposing dark regions, or 'cavities', positioned over atomic vacancies or ion binding sites of hexagonal rings of similar crystal surfaces ${ }^{23-24}$. In addition to site-specific hydration, associated hydration layers with alternating enhanced and dark frequency contrast are typically located further above the surface and similar hydration layers are found on protein surfaces ${ }^{19}$. Allatom MD simulations used to better understand the frequency contrast mechanisms reproduce the 3D FM-AFM experimental observations at the molecular level and show that oscillatory profiles caused by changes in free energy are due to structural rearrangements in the hydration layer manifested through the overlap, confinement and displacement of hydration peaks ${ }^{20-21}$. An overlap of hydration layers on the tip apex atom and sample surface, effectively reforming the intrinsic hydration structures, leads to a decrease in free energy and produces attractive gradients and the energy minima. For the unmodified SiNP, the energy minima in the $1 \mathrm{D}$ profiles correlate to dark regions in the $2 \mathrm{D}$ cross-sections (Figure 2a), with spacings on the order $\approx 0.5 \AA$ occurring in between the structured water layers (i.e. their molecular vdW surfaces). Their occurrence as discrete, interposing 'cavities' within the interfacial water layers also raises interesting questions as to their structural 
significance, including the notion of intermolecular voids that would constitute the free volume between neighbouring water molecules in this region.

\section{D FM-AFM characterisation of hydration layers on GPS-modified high density SiNPs}

The interfacial layer phase of the GPS functionalised SiNPs differs considerably to the unmodified SiNP surface. 3D volume maps reveal a distinct, continuous layer extending to significantly greater distances from the surface, with no evidence of a branched, networklike water structure or interposing 'cavities' (Supplemental Figure S2). 2D cross-sections appear to show the existence of a gradation in the frequency contrast, including initially darker contrast from the bulk water phase followed by an increase in the frequency shift corresponding to the interfacial layer phase that forms a continuous, parallel-aligned layer across the entirety of the GPS modified SiNP (Figure 4a). The change in the frequency shift is consistent with 1D profiles at different $\mathrm{XY}$ positions that only show a purely repulsive profile (Figure 4 a-e). Further analysis by statistically averaging 1D profiles from entire 2D crosssections (ZY-averaged curves) also reveals a more complex s-shaped frequency profile, which is well-described by Boltzmann sigmoid fitting $\left(R^{2}=>0.996\right)$ and consists of a transition in the frequency gradient as defined by the $1^{\text {st }}$ and $2^{\text {nd }}$ derivative functions (Figure 5a-marked by red dotted line). By using a higher frequency setpoint to decrease the distance between the tip and surface, deeper probing into the interfacial layer phase continues to show a repulsive interaction and further accentuates the s-shaped profile. 
Conversion of the frequency shift to a force shows that the original transition in frequency gradient marks the onset of a linear slope region, suggesting constant compliance has been reached subsequent to the deformation of the GPS layer by the AFM tip (Figure 5 (transition marked by dotted lines)). In this case, the zero reference (i.e. the surface) is defined at the onset of constant compliance where effectively the slope of the force curve indicates an incompressible or infinitely hard surface. This is more clearly visualized as the minima or cross-over point in the $1^{\text {st }}$ and $2^{\text {nd }}$ derivative, respectively (Figure $5 \mathbf{a}$ ). Figure $\mathbf{5 b}$ indicate small shifts in the defined zero reference at different $X Y$ positions of the measurement on the SiNP surface. Here, we interpret that the GPS chains have been fully compressed, giving an approximation of the zero reference. The distance from the start of the repulsive interaction until the onset of constant compliance provides an estimate of the interfacial layer thickness, which was measured to be 10-12 (Figure 5). Despite not being able to define a discrete hydration layer, we suggest that the interfacial layer thickness is rationalized by the presence of associated water, which is discussed further below in MD simulations. TGA results indicate that the density of GPS molecules on the $\operatorname{cc} 302{ }^{\circledR} \operatorname{SiNP}$ surface is $\sim 1.52$ molecules. $\mathrm{cm}^{-2}$ (Supplemental Figure 1 ) and at this density one would expect to observe individual GPS chains as opposed to a confluent layer over the $4 \mathrm{~nm}$ scan distance. The interfacial layer may also represent an average, or 'snapshot', of the GPS chain dynamics and associated water molecules that occur on significantly faster timescales than the 3D FM-AFM measurements ${ }^{25}$ thereby experimentally observing all statistical GPS chains positions as a continuous layer. As a result of having a continuous layer, the top-view images taken at various z-slices through the interfacial layer maintains a smooth, more homogenous appearance across the GPS modified SiNP in contrast to the unmodified SiNP (Figure 6). 


\section{Hydration structure of low density GPS-modified SiNP}

To further confirm the interfacial water structuring, a lower density GPS-modified SiNP $\left(\mathrm{cc} 301^{\circ}\right)$ was introduced to observe intermediate effects between the unmodified and highdensity GPS SiNP surfaces (Supplemental Figure 4). 2D cross-sections reveal the continuous layer on the high-density GPS surface, however neighbouring areas just several angstroms away demonstrate more intricate structures that are more representative of water structuring on the unmodified SiNP (Supplemental Figure 4b). Furthermore, in rare cases, discrete angled structures were seen within regions of the GPS-water layer that may represent the unveiling of individual GPS molecules (Supplemental Figure 5). The density of GPS molecules on the $c c 301^{\circledR}\left(0.8\right.$ molecules. $\left.\mathrm{cm}^{-2}\right)$ is therefore not high enough to generate an associated hydrated GPS layer, which is qualitatively commensurate with the mean protein adsorption and level of bacterial and fungal colonisation on these coatings compared to the unmodified and high density GPS SiNP surfaces.

\section{Simulations show experimentally consistent hydration layer structuring on bare and GPS modified silica surfaces}

\section{Unmodified silica}

MD simulations were undertaken to provide a molecular level rationale to the FM-AFM experiments, specifically relating to the population of atomic species at the various 
solid/liquid interfaces. Graphical presentation of the MD simulation data hereafter allows direct comparison and correlation with the 3D FM-AFM data presented in previous sections. 3D atomic density maps were generated from 40 ns MD simulations and normalized by the density of water more than $1.8 \mathrm{~nm}$ from the surface (bulk water) (Figure 7a and b). For the unmodified silica (Figure 7a,b), 2D cross sections were generated and are strikingly similar to the corresponding experimental AFM 2D cross-sectional images. Snapshot images show a predominately single densely, bound water layer (oxygen atoms of water, grey color) closely following the molecular surface topography to give an undulating appearance very similar to the AFM images (Figure 7a). Specifically, at heights less than $0.75 \mathrm{~nm}$ above the silica surface groups, relative density 2D cross-sections confirm that this water layer corresponds to high atomic density regions (red color scale) of individual water molecules located above the unmodified silica substrate (Figure 7b). Low density regions (blue color scale, asterisks) can also be observed in 2D cross sections (Figure 7b), particularly above the regions of high density in the water layer, typically with a height range of between 0.1 and $0.2 \mathrm{~nm}$. Where this pronounced initial water layer is followed by a low-density region, a further increase in the density of water is sometimes observed, indicating a discontinuous second water layer (Figure 7b). The high-density regions are mainly delineated by discrete rounded structures of approximately $1.5-3.0 \AA$ in size, corresponding to dimensions of single water molecules and supporting that the 3D FM-AFM is probing individual water layer(s) above the substrate (Supplementary Figure S6). Furthermore, Supplementary Figure 6 shows that within less than $0.9 \mathrm{~nm}$ from the surface, the height of the first water layer (high density region, red color) can vary by up to $0.5 \mathrm{~nm}$ due to this structured water closely following the atomic roughness of the underlying amorphous substrate, as observed in the 3D FM-AFM.

Supplementary Figure S7 also provides several sequential 2D sections of the relative density 
with accompanying snapshots to emphasize the propagation of the water layer across the unmodified silica surface, including the arrangement of the individual water molecules in the initial layer relative to those involved in an apparent secondary layer. The corresponding simulated averaged 1D density profiles versus distance curves show oscillations with 1-2 peaks (Figure 7c), which is in qualitative agreement with the oscillatory profiles of FM-AFM frequency shift curves and confirms that these arise due to perturbation of up to two intricate, structured water layers.

Simulated $X Y$ contour slices moving from 0.4 to $0.64 \mathrm{~nm}$ above the silica substrate (Figure 7d-f) recapitulate the laterally distributed branched, or network-like, water structure. Due to the heterogeneous distribution of silanol groups and atomic roughness this layer does not form a cooperative or ordered network in the plane parallel to the surface nor is there significant water structuring beyond the first hydration layer. Outside of $0.64 \mathrm{~nm}$ from the substrate $\mathrm{XY}$ slices show water profiles indistinguishable from bulk-like water in line with experimental findings on the short range influence of the substrate on the liquid phase. A snapshot examining water within $0.4 \mathrm{~nm}$ of the unmodified silica (Figure $\mathbf{7 d}, \mathbf{h}$ ) reveals the first hydration layer (red color) covers the entire substrate and is composed of individual water molecules or ions clustered at adsorption sites on the substrate to form the reticulate, network-like structure equivalent to that observed in 2D top-view images of the FM-AFM in Figure 3a(ii).

\section{High density GPS-modified silica}

In line with FM-AFM results, the MD simulations of the high-density GPS modified silica interface show considerable differences from the unmodified silica system. The 3D atomic density map reveals a region of tightly constrained GPS and denser water close to the 
surface $(<0.8 \mathrm{~nm})$ with an associated GPS/less dense water region extending outwards and transitioning into bulk-like water between 0.8-1.2 $\mathrm{nm}$ above the substrate (Figure 8 a). Furthermore, the 2D cross sectional density maps (Figure $\mathbf{8 b}$ ) and 1D density profiles (Figure 8c) indicate this diffuse region has a lack of well-defined hydration layers and void volumes, in contrast to the behaviour observed above the unmodified silica. In this case, distal hydroxyl groups of the GPS have a strong association with water, with an average of 2 hydrogen bonds per ligand from these functional groups. The dynamic nature of the GPS ligands evident in the snapshot image (Figure 8a) means that water molecules engaged in hydrogen bonding with these outer hydroxyl groups are not confined to specific sites on the surface. Corresponding averaged 1D density plots show only a monotonic decrease in bulk water density from 1 to 0 throughout the GPS and associated water region (Figure 8c), confirming that there is no propagation of interfacial water structuring into the bulk solvent. Within the diffuse region, i.e. $0.8-1.2 \mathrm{~nm}$, the average density of the interface remains within $10 \%$ of that of the bulk solvent despite transition from bulk solvent away from the interface to the associated GPS ligand/water mixture (Figure 8c). By considering the height at which the relative density of bulk-like water density falls below 0.5 for each of the 1D profiles, the average thickness of the interface was estimated to be $1.0 \mathrm{~nm}$ (Supplemental Figure S8), which correlates to the estimated value from the experimental force curves, i.e. $10-12 \AA$. In addition, the transition from the diffuse GPS/associated water region to the more confined ligands correlates with the increase in slope of the AFM force profiles in this region $(z<0.8 \mathrm{~nm})$, as shown in Figure 5, and suggested compression of the GPS ligands as the AFM tip to penetrates closer to the surface. 
To support the conclusion that the outer region of the interface $(0.8-1.2 \mathrm{~nm})$ is a diffuse mixture of GPS ligands and water, root mean square fluctuations (RMSFs) for the $C_{6}$ atoms of the GPS ligands were calculated and plotted against $Z$ distance from the surface (Supplemental Figure S9). On average, when above $0.8 \mathrm{~nm}$ from the substrate the fluctuation range of the atom is above $0.3 \mathrm{~nm}$. When found closer to the surface the motion of the free end of the ligand is more limited. Similar trends are observed for the distal hydroxyl oxygen atoms. The time scales on which the RMSF estimates are generated (10s of nanoseconds) also support the hypothesis that the 3D FM-AFM images produce a representative sample (average) of all possible GPS ligand positions.

Sequential simulated 2D XY density maps and associated density distributions ranging from 0.4-1.2 $\mathrm{nm}$ above the surface show the transition from largely segregated GPS and water clusters at Z distances below $0.6 \mathrm{~nm}$ compared to diffuse and overlapping distributions of GPS ligands and water atoms for $Z$ distances between 0.6 and $1.2 \mathrm{~nm}$ above the substrate (Figure 8c). Sequential simulated 2D XY density maps and associated density distributions in this region show increased roughness in line with the experimental observations.

\section{Low density GPS-modified silica}

Deconstruction of the 3D atomic density map produced from MD simulations of the low density GPS system identify regions of the interface that display unmodified and GPS modified silica substrate type profiles (Supplemental Figure S10). 24\% of the interface (327/1352 1D profiles) has a bulk water transition height of less than $0.7 \mathrm{~nm}$ and the average interfacial 1D profile is reminiscent of that for the unmodified silica (Supplementary Figure S10). Similarly, the average of the remaining $36 \%$ of the interface, with a bulk water transition height of greater than $0.7 \mathrm{~nm}$, reflect the behaviour of the GPS modified surface 
(Supplementary Figure 10). GPS ligands in the latter regime are slightly more compressed than the high GPS ligand surface, with an average bulk-like water transition height of 0.88 $\mathrm{nm}$ compared $1.0 \mathrm{~nm}$ for the high density GPS system (Supplementary Figure S8). The regions of exposed substrate likely act as adsorption sites for proteins and larger biological species.

\section{Discussion/Conclusion}

Despite the established role of steric forces in higher molecular weight, longer chain chemistries such as PEG ${ }^{26}$ the contributions from shorter silanes have been a subject of debate for several decades ${ }^{27-29}$ and proposed to play a secondary role to hydration mechanisms ${ }^{30}$. Early work on the nature of repulsive forces originating from short oligo(ethylene glycol)-terminated self-assembled monolayers demonstrate that the forces are unique and thought not to be related to steric repulsion but compatible with proposed binding of interfacial water ${ }^{31}$. Recently, work by the Thompson group shows ultrathin dense adlayers of monoethylene glycol have a high resistance to protein adsorption, with the combination of distal - $\mathrm{OH}$ and ether oxygen -O- groups critical for a putative internal, tightly bound hydration layer ${ }^{32-34}$. They determine short chained organosilanes should possess both internal and external hydrophilicity and komatrophicity to exhibit excellent protein resistant properties, with co-ordination with hydrophilic surface chemistries also a possible contributing factor (34). Furthermore molecular water should be tightly bound with limited lability, presenting a discreet hydration network where the removal of molecular water by approaching proteins would represent an energy penalty, thus resulting in repulsive forces (34). However, further models on hydration layers would significantly benefit from 
experimental evidence that directly visualizes the spatially resolved 2D and 3D molecular structures and interactions of hydrophilic functional groups with the water molecules. There remains an important question as to what is the key structure-function relationship of interfacial water at the short silane protected surfaces that underpins their ultra-low fouling performance as opposed to interfacial water that exists on many other hydrophilic surfaces where protein adsorption is able to persist.

Our study reveals that SiNP-based hydrophilic coatings bearing short chain silane terminal hydroxyl functional groups with different surface configurations have dramatic effects on the interfacial molecular water structuring and properties which we are able to correlate with their antifouling performance. Collectively, the experimental and all-atom modelling approaches support the presence of a continuous, thicker and less dense, GPSassociated water layer on the modified SiNP versus a more tightly bound, dense and stable structured water layer on the unmodified SiNP.

More specifically, the GPS modified SiNP consist of two types of associated water, including 1) more strongly bound and dense water occupying regions of constrained, less dynamic GPS, i.e. at the base of the GPS chains, and 2) less dense, more dynamic water within the outer GPS chains. Our previous MD simulations show that the molecular spacing of the GPS chains enables chain fluctuation dynamics that perturb associated water molecules to create an interfacial regime comprising a dynamic and less dense water layer ${ }^{25}$. Due to significant conformational freedom of the GPS chains, this dynamic interface is not suitable for propagation of interfacial water structuring but rather promotes a diffuse layer with higher mobility water adjacent to the GPS. The dynamic water molecules in the hydration layer are proposed to be capable of competing with the protein for surface 
contact and given sufficient time may rearrange to completely replace the protein at the surface ${ }^{25}$. In addition, the GPS-hydration layer is uninterrupted and prevents exposure of the underlying SiNP, i.e. as opposed to low-density GPS, thus additionally presenting an effective steric barrier.

Related to the interfacial layer structures, the 1D force profiles show the GPS-hydration layer has a purely monotonic repulsion interaction which is fully reversible, i.e. incurs no dissipative losses. Intuitively, this comes with an energy cost for a protein approaching the surface, however reversible desorption is favoured by a decrease in the free energy. For a branched, network-like water structure on the unmodified SiNP, its contrasting 1D oscillatory profile also presents energy barriers but dually consists of energy minima that may increase protein residence times and enable amino acid residues to perturb water layers and undergo binding to the substrate. A comparison of 1D profiles for the different SiNPs confirm that the GPS-modified SiNP have significantly higher repulsive forces and longer-range interactions (Supplemental Figures S11) that are further concomitant with an increased ability of a surface to resist protein adsorption.

Therefore, the 3D FM-AFM uniquely enables correlation of 1D profiles to spatial 2D and 3D distribution of structured water layers, thereby providing experimental data that shows unprecedented convergence to the scale of all-atom simulations performed in this study. From both the experimental and modelling results, we propose that suitably spaced, flexible chains with hydrophilic groups interact with water molecules to produce a continuous quasi-stable, diffuse layer without (hydration) structurally-induced energy minima that provides for effective antifouling performance of ultrathin, hydrophilic surface 
chemistries. This diffuse regime above the GPS modified SiNP surface might exemplify the type of Goldilocks interface described by Voegler et al. at which there is very little difference between the interface and bulk solvent ${ }^{35}$. The contrasting behaviour of the unmodified SiNP gives further insight by enabling comparison with intrinsic, structured water layers that do not prevent protein adsorption and biofouling. Indeed, while the more tightly bound, stable water layers regions on the unmodified SiNP are less likely to rearrange in competition with protein adsorption, as shown by MD simulations ${ }^{25}$, it is intriguing that the interposing dark regions in the AFM images corresponding to simulated low density regions, e.g. excluded volume effects, of the branched, network-like water layer may play a role in facilitating protein adsorption, as previously shown for other systems ${ }^{36}$.

In summary, this study has been undertaken using a common silane agent coupled to SiNP's that are highly processable and easily applied as a coating using spray coating, rollcoating, or 3D printing. We discover these coatings have a broad-ranging fouling resistance from protein adsorption to microbial adhesion of bacteria and fungal spores. By applying a holistic approach whereby the experimental characterisation and theoretical modelling are combined at the same high temporal and spatial resolution, we are able to elucidate the molecular-level interfacial structures to explain the exceptional antifouling ability of practically useful chemistries and coating systems. Therefore, the development of antifouling materials for a multitude of biomedical and industrial applications is significantly advanced. 


\section{Materials and Methods}

\section{Materials}

Three commercially available aqueous $\mathrm{SiO}_{2}$ nanoparticle dispersions were used in this study. Bindzil 30/360 (30wt \%), Bindzil cc301 (30wt \%) and Bindzil cc301 (30wt \%) colloidal silica nanoparticle dispersions were all sourced from Akzonobel, Amsterdam, Netherlands. Hydrophobin SC3 (68795), bovine serum albumin (A-3059), fibrinogen from bovine plasma (F8630) and phosphate buffered saline were all purchased from Sigma Aldrich (Sydney, Australia). Escherichia coli JM109 was a kind gift from Dr Jason McArthur, while Epicoccum nigrum (42773) was purchased from the American Type Culture Collection (ATCC).

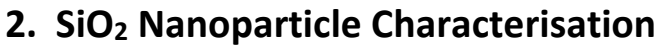

\subsection{Thermogravimetric Analysis}

The degree of surface functionalisation of the various Bindzil ${ }^{\oplus}$ particles was determined using thermogravimetric analysis (TA, Q500). An undiluted aqueous dispersion of Bindzil ${ }^{\bullet}$ $\mathrm{SiO}_{2}$ nanoparticles was first frozen, and then dried in a freeze dryer (Alpha 2-4 LD Plus, Christ, Germany) to remove all water. Dried particles were then heated from room temperature to $800^{\circ} \mathrm{C}$ at a rate of $10^{\circ} \mathrm{C} / \mathrm{min}$ under a nitrogen atmosphere. The mass of surface bound polymer on the particle surface was determined from the weight loss measured between $150^{\circ} \mathrm{C}$ and $800^{\circ} \mathrm{C}$. The remaining mass at $800^{\circ} \mathrm{C}$ was presumed to be that of the bare silica nanoparticles.

\section{Fabrication of $\mathrm{SiO}_{2}$ nanoparticle thin films}


For quartz crystal microbalance (QCM-D) based studies, $\mathrm{SiO}_{2}$ nanoparticle films were fabricated onto A-T cut quartz crystal sensors with a $10 \mathrm{~mm}$ diameter gold electrode and a fundamental resonance frequency of $5 \mathrm{MHz}$ (Q-Sense $A B$ Västra, Frölunda, Sweden). Prior to coating, the QCM-D sensors were cleaned with piranha solution (70\% sulphuric acid and $30 \%$ hydrogen peroxide) for 3 mins, followed by rinsing with deionised water and drying under nitrogen gas. The sensors were then mounted onto a round coverslip and placed into a Bioforce Nanosciences UV/Ozone Procleaner for 20 mins. Thereafter, films were spin coated by depositing $20 \mu \mathrm{l}$ of a $4 \mathrm{wt} \%$ aqueous dispersion of the relevant Bindzil ${ }^{\circledR}$ nanoparticle onto the gold electrode on the sensor, with spin coating performed at 7000 rpm for 30 secs. The films were then cured for 16 hours at a temperature of $110^{\circ} \mathrm{C}$, followed by through rinsing with deionised water and drying under a stream of nitrogen gas. For in-vitro microbial cell adhesion experiments, Bindzil ${ }^{\oplus}$ nanoparticle films were fabricated onto gold coated glass coverslips. ProSciTech 19mm round glass coverslips (G419-5) were cleaned with ethanol, and then coated with $5 \mathrm{~nm}$ titanium and $30 \mathrm{~nm}$ gold using a sputter coater (Edwards Auto 306 thermal evaporator/vacuum coating system). The metal coated coverslips were then placed in a Bioforce Nanosciences UV/Ozone Procleaner for 20 mins, and coated with $\mathrm{Bindzil}^{\oplus} \mathrm{SiO}_{2}$ nanoparticles via spin coating following the same procedure described above for QCM-D sensors.

\section{Film Characterisation}

\subsection{Contact Angle Measurements}


A Dataphysics contact angle system in conjunction with SCA20 software was used to calculate the contact angle of a $2 \mu \mathrm{l}$ sessile drop on the nanoparticle film surfaces. All measurements were performed in triplicate on three samples for each film.

\subsection{Scanning Electron Microscopy}

Scanning electron microscopy (SEM) imaging of the coatings was performed using a field emission SEM (JOEL JSM-7500FA). Samples for SEM analysis were coated onto gold coated QCM-D sensors as described previously. The sensor was then broken into smaller pieces, attached to a SEM mount using silver paint, and imaged without modification of the coating surface. For cross sectional analyses, samples were cracked in half and mounted perpendicular to the surface on a customised sample holder. Thereafter the samples were sputter coated with gold prior to imaging.

\section{QCM-D Protein Adsorption Experiments}

Protein adsorption experiments were performed using a Q-Sense E4 (Q-Sense AB Västra, Frölunda, Sweden) quartz crystal microbalance with dissipation monitoring (QCM-D) in a flow-through setup coupled with an ISMATEC IPC High Precision Multichannel Dispenser (IDEX, Wertheim, Germany) for flow through control. QCM sensors with the various silica nanoparticle coatings were placed in the instrument and equilibrated with PBS for 2 hours at a constant temperature of $22.0^{\circ} \mathrm{C}$. After this time a $1 \mathrm{mg} \cdot \mathrm{mL}^{-1}$ bovine serum albumin (BSA), $1 \mathrm{mg} \cdot \mathrm{mL}^{-1}$ fibrinogen (Fb), or $5 \mu \mathrm{g} \cdot \mathrm{mL}^{-1}$ Hydrophobin SC3 (Hb) in PBS solution was introduced into the chamber at a constant flow rate of $10 \mu \mathrm{L} \cdot \mathrm{min}^{-1}$ for $60 \mathrm{mins}$, after which the chamber was rinsed with PBS for a further 60 minutes at the same flow rate. The 
chamber was maintained at a constant temperature of $22 \pm 0.1{ }^{\circ} \mathrm{C}$ for the entirety of the experiment and each coating was tested in triplicate.

To model the adsorption of both proteins to the coatings, the Q-Sense Qtools data analysis software package was used to apply the Voigt model to determine the mass of the surface bound hydrated protein layer in $\mathrm{ng} . \mathrm{cm}^{-2}$. Input parameters used to best fit the data include a layer density of $1150 \mathrm{~kg} \cdot \mathrm{m}^{-3}$, fluid density of $1020 \mathrm{~kg} \cdot \mathrm{m}^{-3}$, layer viscosity between $10^{-6} \leq 10$

${ }^{2} \mathrm{~kg} \cdot \mathrm{m}^{-1} \mathrm{~s}^{-1}$, layer shear modulus between $10^{4} \leq 10^{8} \mathrm{~Pa}$ and mass between $1.15 \leq 1.15^{5} \mathrm{ng} \cdot \mathrm{cm}^{-}$ 2.

\section{Microbial Adhesion Assays}

\subsection{Cell Culture}

Bacterial solutions were prepared from a pre-cultured JM109 strain of Escherichia coli and inoculated overnight in $5 \mathrm{~mL}$ of sterile LB (Luria-Bertani) medium at $37^{\circ} \mathrm{C}$ in a Bioline incubator shaker 8500 (Edwards Instrument Co., Narellan, Australia). $0.5 \mathrm{~mL}$ of inoculated culture was added to $10 \mathrm{~mL}$ of LB medium and optical density measurements carried out every 30 minutes using a Spectronic 200 (Thermo Scientific, Waltham, MA, USA) until the desired turbidity was achieved $\left(\mathrm{OD}_{600} 0.6-1.0\right)$. The number of colony forming units (CFU) in the bacterial suspension was determined by plating out dilutions of the suspension and was found to be $4.5 \times 10^{6} \mathrm{CFU} / \mathrm{mL}$.

\subsection{Bacterial Adhesion Study}

Coverslips with coatings of Bindzil ${ }^{\circledR}$ nanoparticles were first rinsed in $\mathrm{EtOH}$ and placed into individual wells of sterile 12 -well culture plates. $2 \mathrm{ml}$ of PBS with bacterial cells were added to each well, and culture plates were incubated at $37^{\circ} \mathrm{C}$ for either 24 hours. Coverslips were 
then removed from incubation and placed into fresh sterile 12-well culture plates.

Coverslips were rinsed with $0.7 \% \mathrm{NaCl}$ solution to remove loosely adhered bacteria. This process was repeated three times for each sample. Adhered bacteria were chemically fixed by depositing $0.5 \mathrm{~mL}$ of $3.7 \%$ paraformaldehyde in PBS solution onto the surface of each sample.

\subsection{Bacterial Staining and Imaging}

Samples were stained with a $20 \mu \mathrm{g} / \mathrm{mL}$ Hoechst 33342, trihydrochloride, trihydrate working solution (Invitrogen - Life Technologies (Thermo Scientific)). $1 \mathrm{~mL}$ of the Hoechst working solution was deposited onto each of the coated QCM sensors and left for 15 minutes. Stained bacteria were imaged using a Zeiss Axiolmager A1M with an open HBO 100 mercury lamp and an Axiocam MRm camera (Carl Zeiss, Oberkochen, Germany). Images were obtained at 50x magnification, with bacterial cell counts determined using Image ${ }^{\circledR}$ software (v.1.50b).

\section{Fungal Spore Adhesion Study}

\subsection{Adhesion Assay}

The antifouling properties of the Bindzil ${ }^{\circledR}$ nanoparticle coatings were evaluated against Epicoccum nigrum (ATCC 42773). E. nigrum spores were collected from a pre-cultured agar plate (Potato dextrose agar (BD 213400)) and dispersed in $7 \mathrm{~mL}$ sterile water. The spore suspension was shaken vigorously and placed on an orbital shaker for 2 hours. The suspension was then centrifuged for 5 minutes at 1500 RPM. The supernatant was removed and spores were resuspended in $7 \mathrm{~mL}$ sterile water. The spore suspension was then filtered 
through a $40 \mu \mathrm{m}$ hollander weave mesh to ensure good spore separation and diluted $~ 10-$ fold.

Spin-coated nanoparticle coatings were placed into a sterile Greiner 12 well cell culture plate and covered with $1 \mathrm{~mL}$ sterile deionized water. An aliquot of spore suspension was then deposited onto coatings to give a concentration of $6 \times 10^{3}$ spores per $\mathrm{cm}^{2}$ and samples were incubated at $30^{\circ} \mathrm{C}$ for 24 hours. Samples were removed from the incubator after 24 hours and each coverslip was immersed in sterile water $(3 x)$ with gentle agitation, then washed $(3 x)$ with sterile water. Spores were fixed with $2.5 \%(v / v)$ glutaraldehyde solution.

\subsection{Cell Imaging}

Fixed spores were imaged at 5x magnification using a Zeiss Axiovert inverted microscope. Areas imaged were chosen randomly and spores counted using Image ${ }^{\oplus}$ software (v.1.50b). 4 images were obtained for each sample surface and each surface was sampled in triplicate with the total average reported.

\section{Statistical Analysis}

Numerical results were expressed as means $\pm 95 \%$ confidence intervals. Results were analyzed using one-way analysis of variance (ANOVA) with Tukey post hoc test. Probabilities of $p<0.05$ were considered to be significantly different. All statistical analyses were performed using MINITAB 18 statistical software. 


\section{FM-AFM Experiments}

\subsection{D FM-AFM Measurement}

The experiments were performed with a home-built frequency modulation atomic force microscopy (FM-AFM). The physical and pixel sizes of the original 3D $\Delta \mathrm{f}$ images for Bindzil $30 / 360$ and CC301 nanoparticle surfaces were $4 \times 4 \times 2 \mathrm{~nm}^{3} 64 \times 64 \times 256$ pixels, and for Bindzil $^{\oplus}$ CC302 nanoparticle surface were $4 \times 4 \times 3 \mathrm{~nm}^{3}$ and $64 \times 64 \times 256$ pixels. The frequency and amplitude of the $z$ modulation and lateral scan speed during the FM-AFM imaging were $195.3 \mathrm{~Hz}, 2 \mathrm{~nm}$ (Bindzil ${ }^{\oplus}$ 30/360 and CC301) or $3 \mathrm{~nm}$ (Bindzil ${ }^{\circledR}$ CC302), and 12.2 $\mathrm{nm} \mathrm{s}^{-1}$, respectively.

\subsection{Microcantilevers}

AC 55 (Olympus) cantilevers were coated with Si (30nm) using a dc Sputter coater (K575XD, Emitech) to remove the contaminants. The resonant frequency $\left(f_{0}\right)$ and quality factors $(Q)$ and spring constant $(k)$ of the cantilevers in $10 \mathrm{mM} \mathrm{NaCl}$ were in the following range, $\mathrm{f}_{0}=$ $780-1250 \mathrm{kHz}, \mathrm{Q}=8-12$ and $\mathrm{k}=40-115 \mathrm{~N} / \mathrm{m}$.

\subsection{Sample Preparation}

For FM-AFM based studies, $\mathrm{SiO}_{2}$ nanoparticle films were fabricated onto cover slips with the diameter as $12 \mathrm{~mm}$. Prior to coating, the glass cover slips were cleaned with ethanol, followed by rinsing with deionized water and drying under nitrogen gas. The cleaned cover slips were then placed into a Bioforce Nanosciences UV/Ozone Procleaner for 20 mins. Thereafter, the cover slips were incubated in $0.33 \mathrm{wt} \%$ poly (ethyleneimine) (PEI) solution for $5 \mathrm{~min}$ then rinsed thoroughly with deionized water, and dried in nitrogen gas. The nanoparticle films were spin-coated twice onto the treated cover slips by depositing $40 \mu \mathrm{l}$ 
and $20 \mu \mathrm{l}$ of a $6 \mathrm{wt} \%$ aqueous dispersion of the relevant Bindzil ${ }^{\oplus}$ nanoparticle at $7000 \mathrm{rpm}$ for 30 secs. The coatings were then cured for 1 hour at $120^{\circ} \mathrm{C}$ and followed by thoroughly rinsing with deionized water and drying under a stream of nitrogen gas. $10 \mathrm{mM} \mathrm{NaCl}$ solution was prepared with Milli-Q water.

The cover slips were glued onto a sample holder. Prior to the FM-AFM measurement, the nanoparticle films were thoroughly rinsed with Milli-Q water and dried under nitrogen gas. Thereafter, $100 \mu \mathrm{l}$ of $10 \mathrm{mM} \mathrm{NaCl}$ solution was dropped onto the sample surfaces.

\subsection{Model details}

We employed a previously validated silica surface model ${ }^{37-38}$ originated from Feuston et al. ${ }^{39}$ This represents a realistic, highly hydrated amorphous silica surface with a surface silanol density of $4.7 \mathrm{OH}$ groups per $\mathrm{nm}^{2}{ }^{40}$ The amorphous silica substrate displays a density of 2.6 $\mathrm{g} / \mathrm{cm}^{3}$ (comparable to experiment), an average film thickness of $1.7 \mathrm{~nm}$ (in the $z$ direction) and lateral dimensions of $2.7 \times 2.7 \mathrm{~nm}^{2}$. Hydrolysed glycidoxypropylsilane (GPS) were added to the bare silica surface replacing surface hydroxyl groups as described previously. ${ }^{41-46}$ Grafted chain density was kept consistent with experiment on both sides of the silica substrate with a chain densities of 1.5 chains $/ \mathrm{nm}^{2}$ (11 total GPS ligands) and 0.9 chains $/ \mathrm{nm}^{2}$ (7 total GPS ligands) for the high and low modified SiNP respectively. The influence of surface charge on the GPS ligands was accounted for by performing simulations at three different surface charge densities: 0, 14\% (5 total) and 28\% (10 total) hydroxyls deprotonated for the high density GPS modified silica system. Partial atomic charges for substrate atoms are shown in Supplementary Table 1. The systems were subsequently hydrated with approximately 800 water molecules on both side of the substrate with additional sodium ions to counter surface charge. During the subsequent simulations, the 
surface silanol groups remained free to move, while the underlying $\mathrm{SiO}_{2}$ atoms were kept fixed. All data presented is for the $14 \%$ deprotonated surface except where specified.

A second larger system was constructed by replicating the unit cell in the $\mathrm{x}$ and $\mathrm{y}$ dimension to give lateral dimensions of $5.4 \times 5.4 \mathrm{~nm}^{2}$. Grafted chain density was for high and low systems were 1.5 chains $/ \mathrm{nm}^{2}$ ( 44 total GPS ligands) and 0.75 chains $/ \mathrm{nm}^{2}$ ( 22 total GPS ligands) GPS modified SiNP respectively. Surface charge was accounted for by deprotonating $20 \%$ (28 total) of surface hydroxyls deprotonated. The systems were subsequently hydrated with approximately 5000 water molecules in the unit cell and $\mathrm{NaCL}$ at $0.1 \mathrm{M}$ with additional sodium ions to counter surface charge. During the subsequent simulations, the surface silanol groups remained free to move, while the underlying $\mathrm{SiO}_{2}$ atoms were kept fixed.

\subsection{Simulation Setup}

The COMPASS force field ${ }^{47}$ optimised for the simulation of condensed phase polymers and organic/inorganic interfaces was used to evaluate the inter- and intramolecular interactions within the all-atom models. Energy minimisation was performed to relieve any induced strain in the constructed models prior to MD simulations. For energy minimisation, nonbonded interactions were calculated using the Ewald procedure with and accuracy of 0.01 kcal mol-1 and an update width of $1.0 \AA$. The conjugate gradient algorithm was used for energy minimisation, with an energy convergence criterion of $0.01 \mathrm{kcal} \mathrm{mol}-1 \AA^{-1}$. For MD procedures, non-bonded interactions were calculated using the atom-based summation method with a cut-off radius of $12.5 \AA$, a spline width of $2.5 \AA$ and a buffer width of $1.0 \AA$. . A long-range vdW tail correction was applied for nonbonded interactions larger than the cutoff radius. A 1.0-fs time step was used for the simulation of the NVT ensemble, utilising the Andersen thermostat ${ }^{48}$ to control the temperature with a collision ratio of 1.0. All runs were 
performed for 42 million timesteps with statistics generated over the final 40 million timesteps (40 ns) with system coordinates being output every 2 ps.

The second set of simulations performed with the larger system model were performed using molecular dynamics (MD) simulation as implemented in $\mathrm{NAMD}^{49}$ was used for all the work reported here. An all-atom representation of the ligands and substrate was used with the intra- and inter-molecular interactions being modelled by the CGenFF 36 potential ${ }^{50}$ with additional parameters taken from existing literature for the silica substrate ${ }^{51}$ and siloxane bond..$^{52}$ Water molecules were treated explicitly using the TIP3P water model. ${ }^{53}$ In all simulations, a cut-off distance of $12 \AA$ was applied for non-bonded interactions with switching applied between 10 and $12 \AA$. Long range electrostatic interactions were treated using the particle mesh Ewald method. ${ }^{54} \mathrm{~A}$ timestep of $2 \mathrm{fs}$ was used in all simulations; this was made possible by fixing the $\mathrm{O}-\mathrm{H}$ bond lengths of the water molecules using the SHAKE algorithm. ${ }^{55}$ Simulations were undertaken using a Langevin thermostat ${ }^{56}$ with a damping coefficient of $1 \mathrm{ps}^{-1}$ to control the temperature. All runs were performed for 60 million timesteps with statistics generated over the final 50 million timesteps (100 ns) with system coordinates being output every 5000 time steps. Data from this simulation is presented in Supplementary Figures 19 to 24.

\subsection{D density profile generation}

3D atomic density profiles were generated from the 50 ns MD simulations for each system. The two $2.6 \times 2.6 \times 2 \mathrm{~nm}^{3}$ interfacial areas (top and bottom sides of the substrate) were separated into an $[x, y, z]$ arrays of $[26,26,100]$ bins each with dimensions $0.1 \mathrm{~nm} \times 0.1 \mathrm{~nm} x$ $0.02 \mathrm{~nm}$, or a volume of $0.0002 \mathrm{~nm}^{3}$. The first bin in the $z$ direction was defined as between 
0 and $0.02 \mathrm{~nm}$ above the average position of Si atoms bonded to surface silanol groups. For the unmodified silica three selections were defined: (1) oxygen atoms from water molecules within $0.35 \mathrm{~nm}$ of the substrate; (2) oxygen atoms from water molecules not within $0.35 \mathrm{~nm}$ of the substrate and ( 3 ) sodium atoms: and for the time points (10000 total) the positions of each atoms was recorded in one of the [ $26 \times 26 \times 100]$ bins. The density of each bin was then normalised with reference to the average density of the 13520 bins in the $1.8-2.0 \mathrm{~nm}$ height range. An identical procedure was done for the larger system creating [ $50 \times 50 \times 100]$ array.

\section{Acknowledgements}

The authors would like to thank the Australian National Nanofabrication Facility materials node for equipment use and the Australian Research Council for funding through the Industrial Transformation Research Hub (IH130100017) and the ARC Centre of Excellence for Electromaterials Science (CE140100012). The authors acknowledge use of the facilities and the assistance of Dr. Tony Romeo at the UOW Electron Microscopy Centre. This research has been conducted with the support of the Australian Government Research Training Program Scholarship and M.J.H. acknowledges an Australian Research Council Fellowship (DP110104359). We also acknowledge the generous allocation of high performance computational resources from the Australian National Computational Infrastructure ( $\mathrm{NCl})$, the Western Australian computational facility (iVEC), the Victorian Partnership for Advanced Computing (VPAC) and the Victorian Life Sciences Computational Initiative (VLSCI). 


\section{References}

1. Scott, R. D., The direct medical costs of healthcare-associated infections in US hospitals and the benefits of prevention. 2009.

2. Klevens, R. M.; Edwards, J. R.; Richards Jr, C. L.; Horan, T. C.; Gaynes, R. P.; Pollock, D. A.; Cardo, D. M., Estimating health care-associated infections and deaths in US hospitals, 2002. Public Health Rep. 2007, 122 (2), 160-166.

3. Schultz, M. P.; Bendick, J. A.; Holm, E. R.; Hertel, W. M., Economic impact of biofouling on a naval surface ship. Biofouling 2011, 27 (1), 87-98.

4. $\quad$ Endresen, $\varnothing$.; Dals $\varnothing$ ren, S.; Eide, M.; Isaksen, I. S.; Sørgård, E. In The environmental impacts of increased international maritime shipping, past trends and future perspectives, Proceedings of the Global Forum on Transport and Environment in a Globalising World, Guadalajara, Mexico, November, 2008; pp 10-12.

5. Organization, I. M., International convention on the control of harmful anti-fouling systems on ships. International Marine Organization London, UK: 2001.

6. Magin, C. M.; Cooper, S. P.; Brennan, A. B., Non-toxic antifouling strategies. Mater. Today 2010, 13 (4), 36-44.

7. Chen, S.; Li, L.; Zhao, C.; Zheng, J., Surface hydration: Principles and applications toward lowfouling/nonfouling biomaterials. Polymer 2010, 51 (23), 5283-5293.

8. He, Y.; Chang, Y.; Hower, J. C.; Zheng, J.; Chen, S.; Jiang, S., Origin of repulsive force and structure/dynamics of interfacial water in OEG-protein interactions: a molecular simulation study. PCCP 2008, 10 (36), 5539-5544.

9. Chu, L.; Daniels, M. W.; Francis, L. F., Use of (Glycidoxypropyl)trimethoxysilane as a Binder in Colloidal Silica Coatings. Chem. Mater. 1997, 9 (11), 2577-2582.

10. Horr, T. J.; Reynolds, G. D., The reactions of 3-glycidoxypropyltrimethoxysilane in acidic solutions on polymerization and in the presence of silica. J. Adhes. Sci. Technol. 1997, 11 (7), 9951009.

11. Innocenzi, P.; Figus, C.; Kidchob, T.; Valentini, M.; Alonso, B.; Takahashi, M., Sol-gel reactions of 3-glycidoxypropyltrimethoxysilane in a highly basic aqueous solution. Dalton Transactions 2009, (42), 9146-9152.

12. Fukuma, T.; Ueda, Y.; Yoshioka, S.; Asakawa, H., Atomic-scale distribution of water molecules at the mica-water interface visualized by three-dimensional scanning force microscopy. Phys. Rev.

Lett. 2010, 104 (1), 016101.

13. Fukuma, T., Water distribution at solid/liquid interfaces visualized by frequency modulation atomic force microscopy. Science and technology of advanced materials 2010, 11 (3), 033003.

14. Albrecht, T. R.; Grütter, P.; Horne, D.; Rugar, D., Frequency modulation detection using high - Q cantilevers for enhanced force microscope sensitivity. J. Appl. Phys. 1991, 69 (2), 668-673.

15. Fukuma, T.; Kobayashi, K.; Matsushige, K.; Yamada, H., True molecular resolution in liquid by frequency-modulation atomic force microscopy. Appl. Phys. Lett. 2005, 86 (19), 193108.

16. Higgins, M. J.; Polcik, M.; Fukuma, T.; Sader, J. E.; Nakayama, Y.; Jarvis, S. P., Structured Water Layers Adjacent to Biological Membranes. Biophys. J. 2006, 91 (7), 2532-2542.

17. Fukuma, T.; Higgins, M. J.; Jarvis, S. P., Direct Imaging of Individual Intrinsic Hydration Layers on Lipid Bilayers at Ångstrom Resolution. Biophys. J. 2007, 92 (10), 3603-3609.

18. Fukuma, T.; Higgins, M. J.; Jarvis, S. P., Direct Imaging of Lipid-Ion Network Formation under Physiological Conditions by Frequency Modulation Atomic Force Microscopy. Phys. Rev. Lett. 2007, 98 (10), 106101.

19. Herruzo, E. T.; Asakawa, H.; Fukuma, T.; Garcia, R., Three-dimensional quantitative force maps in liquid with 10 piconewton, angstrom and sub-minute resolutions. Nanoscale 2013, 5 (7), 2678-2685. 
20. Fukuma, T.; Reischl, B.; Kobayashi, N.; Spijker, P.; Canova, F. F.; Miyazawa, K.; Foster, A. S., Mechanism of atomic force microscopy imaging of three-dimensional hydration structures at a solidliquid interface. Physical Review B 2015, 92 (15), 155412.

21. Miyazawa, K.; Kobayashi, N.; Watkins, M.; Shluger, A. L.; Amano, K.-i.; Fukuma, T., A relationship between three-dimensional surface hydration structures and force distribution measured by atomic force microscopy. Nanoscale 2016, 8 (13), 7334-7342.

22. Israelachvili, J. N., Intermolecular and surface forces. Academic press: 2015.

23. Miyazawa, K.; Watkins, M.; Shluger, A. L.; Fukuma, T., Influence of ions on two-dimensional and three-dimensional atomic force microscopy at fluorite-water interfaces. Nanotechnology 2017, 28 (24), 245701.

24. Tracey, J.; Miyazawa, K.; Spijker, P.; Miyata, K.; Reischl, B.; Canova, F. F.; Rohl, A. L.; Fukuma, T.; Foster, A. S., Understanding $2 \mathrm{D}$ atomic resolution imaging of the calcite surface in water by frequency modulation atomic force microscopy. Nanotechnology 2016, 27 (41), 415709.

25. Penna, M.; Ley, K.; Maclaughlin, S.; Yarovsky, I., Surface heterogeneity: a friend or foe of protein adsorption - insights from theoretical simulations. Faraday Discuss. 2016, 191 (0), 435-464.

26. Kingshott, P.; Thissen, H.; Griesser, H. J., Effects of cloud-point grafting, chain length, and density of PEG layers on competitive adsorption of ocular proteins. Biomaterials 2002, 23 (9), 20432056.

27. Israelachvili, J. N.; Wennerstroem, H., Hydration or steric forces between amphiphilic surfaces? Langmuir 1990, 6 (4), 873-876.

28. Parsegian, V. A.; Zemb, T., Hydration forces: Observations, explanations, expectations, questions. Current Opinion in Colloid \& Interface Science 2011, 16 (6), 618-624.

29. Valle-Delgado, J.; Molina-Bolivar, J.; Galisteo-Gonzalez, F.; Galvez-Ruiz, M.; Feiler, A.; Rutland, M. W., Hydration forces between silica surfaces: Experimental data and predictions from different theories. The Journal of chemical physics 2005, 123 (3), 034708.

30. Harder, P.; Grunze, M.; Dahint, R.; Whitesides, G. M.; Laibinis, P. E., Molecular Conformation in Oligo(ethylene glycol)-Terminated Self-Assembled Monolayers on Gold and Silver Surfaces Determines Their Ability To Resist Protein Adsorption. The Journal of Physical Chemistry B 1998, 102 (2), 426-436.

31. Feldman, K.; Hähner, G.; Spencer, N. D.; Harder, P.; Grunze, M., Probing Resistance to Protein Adsorption of Oligo(ethylene glycol)-Terminated Self-Assembled Monolayers by Scanning Force Microscopy. J. Am. Chem. Soc. 1999, 121 (43), 10134-10141.

32. Blaszykowski, C.; Sheikh, S.; Thompson, M., Surface chemistry to minimize fouling from blood-based fluids. Chem. Soc. Rev. 2012, 41 (17), 5599-5612.

33. Sheikh, S.; Yang, D. Y.; Blaszykowski, C.; Thompson, M., Single ether group in a glycol-based ultra-thin layer prevents surface fouling from undiluted serum. Chem. Commun. 2012, 48 (9), 13051307.

34. Sheikh, S.; Blaszykowski, C.; Nolan, R.; Thompson, D.; Thompson, M., On the hydration of subnanometric antifouling organosilane adlayers: A molecular dynamics simulation. J. Colloid Interface Sci. 2015, 437, 197-204.

35. Vogler, E. A., The Goldilocks Surface. Biomaterials 2011, 32 (28), 6670-6675.

36. Penna, M. J.; Mijajlovic, M.; Biggs, M. J., Molecular-Level Understanding of Protein Adsorption at the Interface between Water and a Strongly Interacting Uncharged Solid Surface. J. Am. Chem. Soc. 2014, 136 (14), 5323-5331.

37. Henry, D. J.; Evans, E.; Yarovsky, I., Classical Molecular Dynamics Study of [60]Fullerene Interactions with Silica and Polyester Surfaces. The Journal of Physical Chemistry B 2006, 110 (32), 15963-15972.

38. Yarovsky, I.; Aguilar, M.-I.; Hearn, M. T. W., Influence of the Chain Length and Surface Density on the Conformation and Mobility of n-Alkyl Ligands Chemically Immobilized onto a Silica Surface. Anal. Chem. 1995, 67 (13), 2145-2153. 
39. Topological and bonding defects in vitreous silica surfaces. The Journal of Chemical Physics 1989, $91(1)$, 564-570.

40. Boehm, H.-P., The Chemistry of Silica. Solubility, Polymerization, Colloid and Surface Properties, and Biochemistry. Von R. K. Iler. John Wiley and Sons, Chichester 1979. XXIV, 886 S., geb. f 39.50. Angew. Chem. 1980, 92 (4), 328-328.

41. Yiapanis, G.; Henry, D. J.; Evans, E.; Yarovsky, I., Simulations of Nanoindentation of Polymer Surfaces: Effects of Surface Cross-Linking on Adhesion and Hardness. The Journal of Physical Chemistry C 2010, 114 (1), 478-486.

42. Yiapanis, G.; Henry, D. J.; Maclaughlin, S.; Evans, E.; Yarovsky, I., Effect of Substrate on the Mechanical Response and Adhesion of PEGylated Surfaces: Insights from All-Atom Simulations. Langmuir 2012, 28 (50), 17263-17272.

43. Yiapanis, G.; Maclaughlin, S.; Evans, E. J.; Yarovsky, I., Nanoscale Wetting and Fouling Resistance of Functionalized Surfaces: A Computational Approach. Langmuir 2014, 30 (35), 1061710625.

44. Yiapanis, G.; Henry, D. J.; Evans, E.; Yarovsky, I., Effect of Aging on Interfacial Adhesion between Polyester and Carbon-Based Particles: A Classical Molecular Dynamics Study. The Journal of Physical Chemistry C 2007, 111 (17), 6465-6472.

45. Yiapanis, G.; Henry, D. J.; Evans, E.; Yarovsky, I., Effect of Surface Composition and Atomic Roughness on Interfacial Adhesion between Polyester and Amorphous Carbon. The Journal of Physical Chemistry C 2007, 111 (7), 3000-3009.

46. Yiapanis, G.; Henry, D. J.; Evans, E.; Yarovsky, I., Molecular Dynamics Study of Polyester Surfaces and Fullerene Particles in Aqueous Environment. The Journal of Physical Chemistry C 2008, 112 (46), 18141-18149.

47. Sun, H., COMPASS: An ab Initio Force-Field Optimized for Condensed-Phase ApplicationsOverview with Details on Alkane and Benzene Compounds. The Journal of Physical Chemistry B 1998, 102 (38), 7338-7364.

48. Molecular dynamics simulations at constant pressure and/or temperature. The Journal of Chemical Physics 1980, 72 (4), 2384-2393.

49. Phillips, J. C.; Braun, R.; Wang, W.; Gumbart, J.; Tajkhorshid, E.; Villa, E.; Chipot, C.; Skeel, R. D.; Kalé, L.; Schulten, K., Scalable molecular dynamics with NAMD. J. Comput. Chem. 2005, 26 (16), 1781-1802.

50. Vanommeslaeghe, K.; Hatcher, E.; Acharya, C.; Kundu, S.; Zhong, S.; Shim, J.; Darian, E.; Guvench, O.; Lopes, P.; Vorobyov, I.; Mackerell, A. D., CHARMM general force field: A force field for drug-like molecules compatible with the CHARMM all-atom additive biological force fields. $J$. Comput. Chem. 2010, 31 (4), 671-690.

51. Butenuth, A.; Moras, G.; Schneider, J.; Koleini, M.; Köppen, S.; Meißner, R.; Wright, L. B.; Walsh, T. R.; Ciacchi, L. C., Ab initio derived force-field parameters for molecular dynamics simulations of deprotonated amorphous-SiO2/water interfaces. physica status solidi (b) 2012, 249 (2), 292-305.

52. Bahar, I.; Zuniga, I.; Dodge, R.; Mattice, W. L., Conformational statistics of poly(dimethylsiloxane). 1. Probability distribution of rotational isomers from molecular dynamics simulations. Macromolecules 1991, 24 (10), 2986-2992.

53. Comparison of simple potential functions for simulating liquid water. The Journal of Chemical Physics 1983, 79 (2), 926-935.

54. Particle mesh Ewald: An $\mathrm{N} \cdot \log (\mathrm{N})$ method for Ewald sums in large systems. The Journal of Chemical Physics 1993, 98 (12), 10089-10092.

55. Ryckaert, J.-P.; Ciccotti, G.; Berendsen, H. J. C., Numerical integration of the cartesian equations of motion of a system with constraints: molecular dynamics of n-alkanes. Journal of Computational Physics 1977, 23 (3), 327-341.

56. McQuarrie, D. A., Statistical mechanics. Harper \& Row: New York, 1975. 

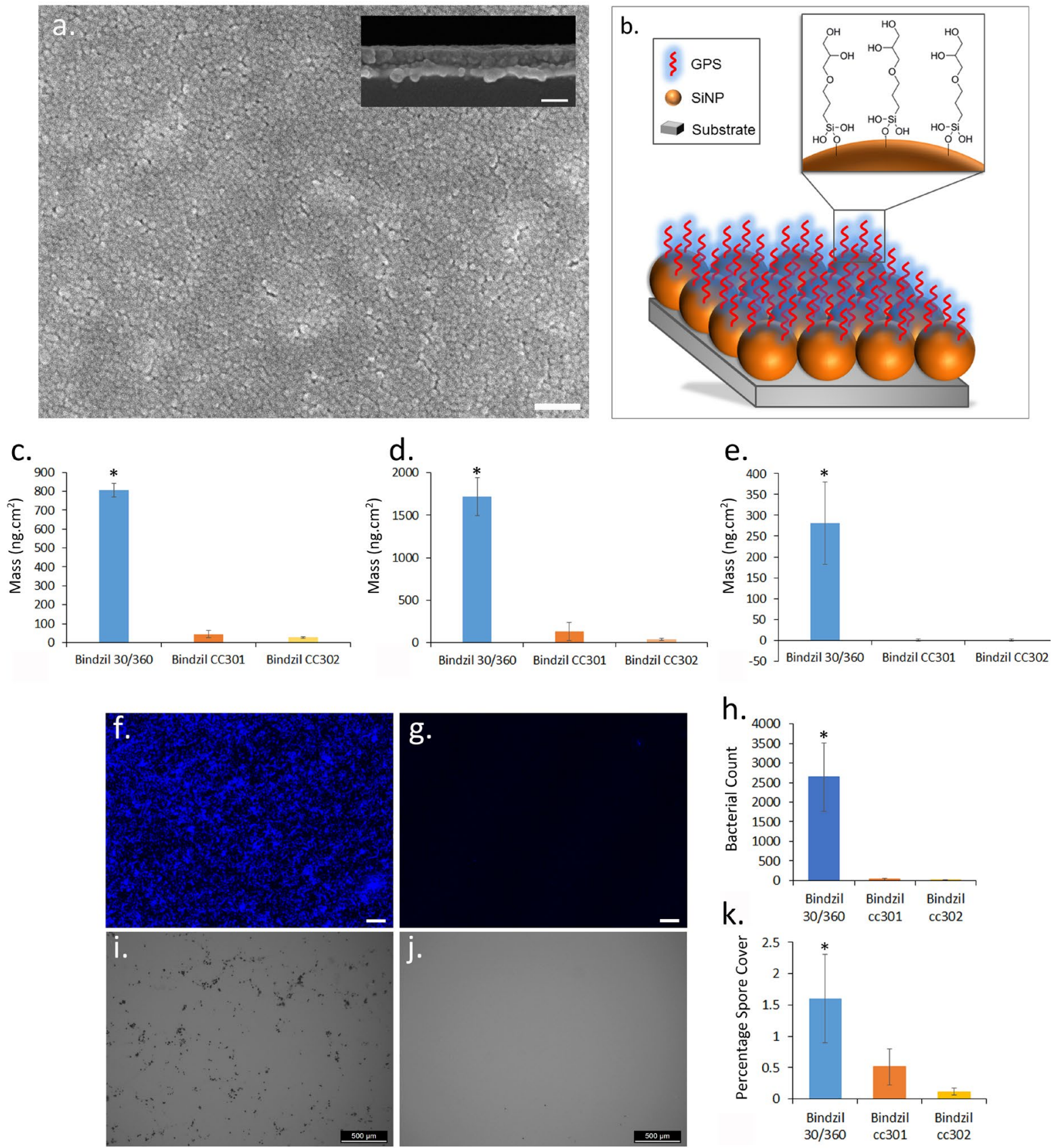

Figure 1: a. SEM image of the surface and cross section (inset) of Bindzil 30/360 modified QCM-D sensor surface (both scale bars are $100 \mathrm{~nm}$ ). b. Schematic of GPS chemical functionality on Bindzil cc $301^{\circ}$ and $\mathrm{cc} 302^{\circ} \mathrm{SiO}_{2}$ nanoparticles. c-e. QCM-D measurements of the mass of the proteins bovine serum albumin (c), fibrinogen (d) and hydrophobin (e) adsorbed to $\mathrm{Bindzil}^{\oplus} \mathrm{SiO}_{2}$ nanoparticle modified sensors. $\mathrm{f}$-g. Representative images of $E$. coli adhesion to Bindzil $30 / 360^{\circ}(\mathrm{f})$ and Bindzil $\operatorname{cc} 302^{\circ}$ (g). h. Mean number of $E$. coli adhered to Bindzil $30 / 360^{\circ}$, Bindzil cc301 ${ }^{\circ}$ and Bindzil $c c 302^{\circ}$ coatings. i-j. Representative images of Epicoccum nigrum adhesion to Bindzil $30 / 360^{\circ}$ (i) and Bindzil $c c 302^{\circ}$ (j). k. Mean number of Epicoccum nigrum spores adhered to Bindzil $30 / 360^{\circ}$, Bindzil cc $301^{\circ}$ and Bindzil cc $302^{\circ}$ coatings. All error bars represent $95 \%$ confidence intervals around the mean. ${ }^{*}$ represents significant difference between the indicated sample and all other samples $(p<0.05)$. 

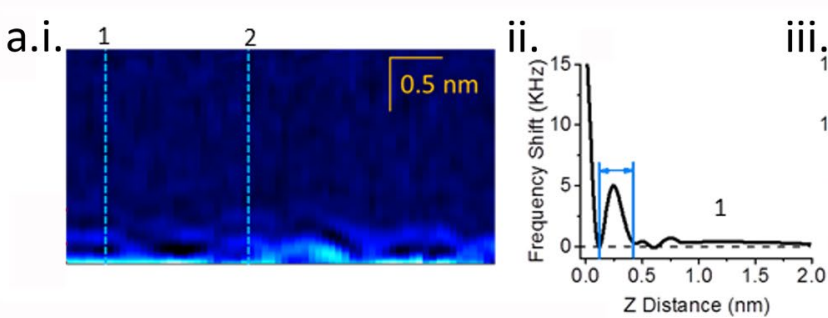

b.
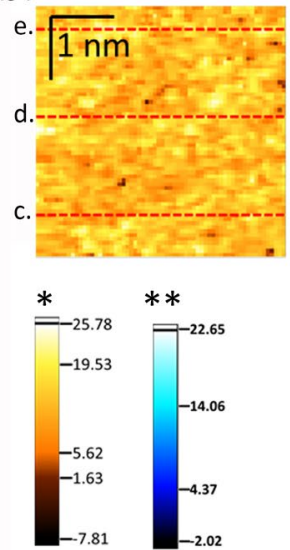

c.i.

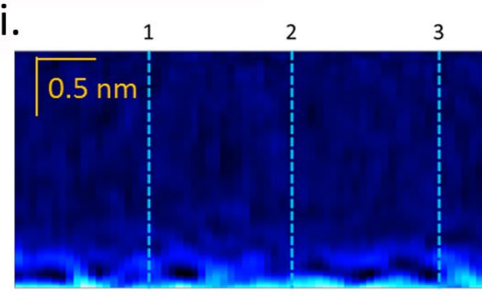

d.i.1

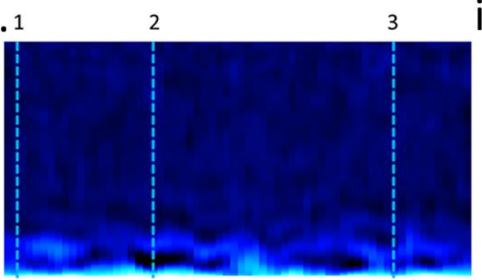

e.i.

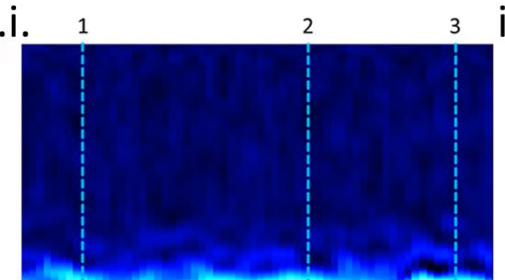

3 ii

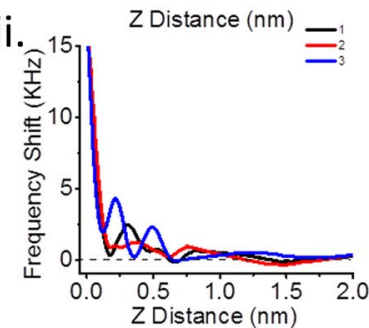

ii.

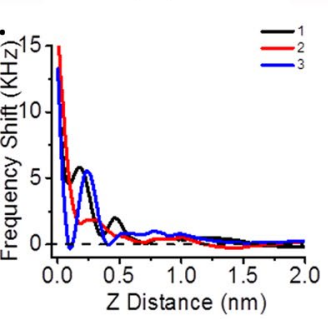

ii.

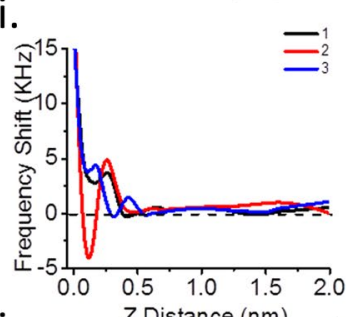

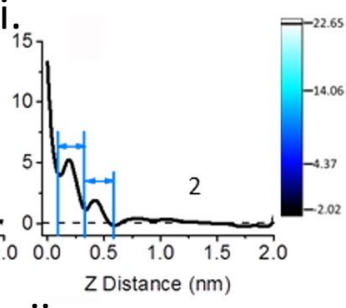

f.i.

ii. After Height Correction

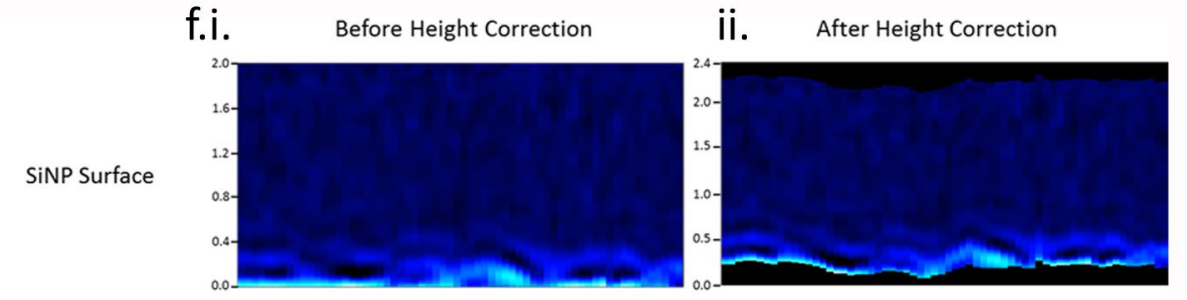

Figure 2. a. Representative $2 D$ cross section at the SiNP interface (i), with representative 1D profiles taken at positions 1 (ii) and 2 (iii) on the SiNP surface. b. 2D top-view image illustrating positions of cross sections and 1D profiles across a SiNP surface. Scanning area is $4 \mathrm{~nm} * 4 \mathrm{~nm} * 2 \mathrm{~nm}$. The 2D top view image is taken at $\mathrm{z}=0 \mathrm{~nm}$ and labelled with dotted lines $(a-c)$ at the position of each 2D cross section (c.i., d.i., e.i.), which are also labelled with positions of 1D profiles taken at positions 1,2 and 3 that are labelled with black, red and blue lines in the 1D profiles, respectively (c.ii., d.ii., e.ii.). f. Before height correction (i) and after height correction (ii) 2D sections. Legend: * illustrates relative frequency difference for $b .{ }^{* *}$ illustrates relative frequency difference for $c, d, e \& f$. The cantilever parameters: $A$ $=1.79 \AA, \mathrm{fO}=956 \mathrm{kHz}, \mathrm{Q}=10.6$ and $\mathrm{k}=45.76 \mathrm{~N} / \mathrm{m}$. The 3D $\Delta \mathrm{f}$ images parameters: The 
frequency and amplitude of the $z$ modulation during the 3D-SFM imaging were $195.3 \mathrm{~Hz}, 2$ $\mathrm{nm}$, and $12.2 \mathrm{~nm} \mathrm{~s}-1$, respectively.

a.

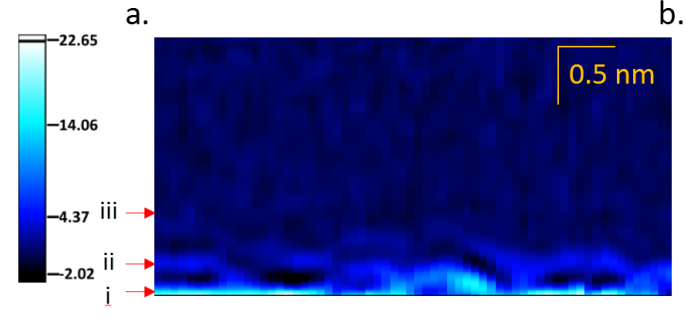

C.

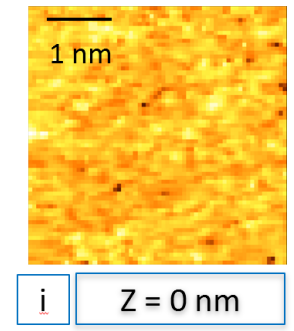

b.

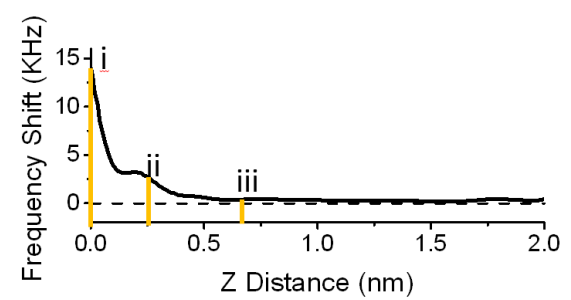

Z Distance $(\mathrm{nm})$
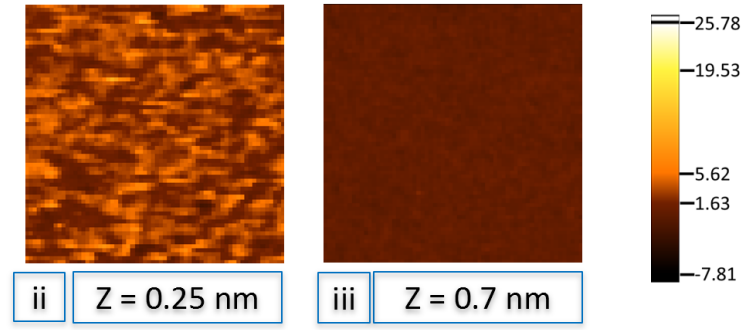

Figure 3: a. Representative 2D cross section at the SiNP interface with positions identified where 2D x-y cross sections ( $\mathrm{i}-\mathrm{iii}$ ) were taken for (b) and (c). b. Averaged 1D frequency shift vs distance curves. c. i - iii. Representative $2 \mathrm{D}$ cross sections taken at points $0 \mathrm{~nm}$ (i), 0.25 $\mathrm{nm}$ (ii) and $0.7 \mathrm{~nm}$ (iii) from the surface (points highlighted on 1D frequency shift vs. distance curves in (a)). 

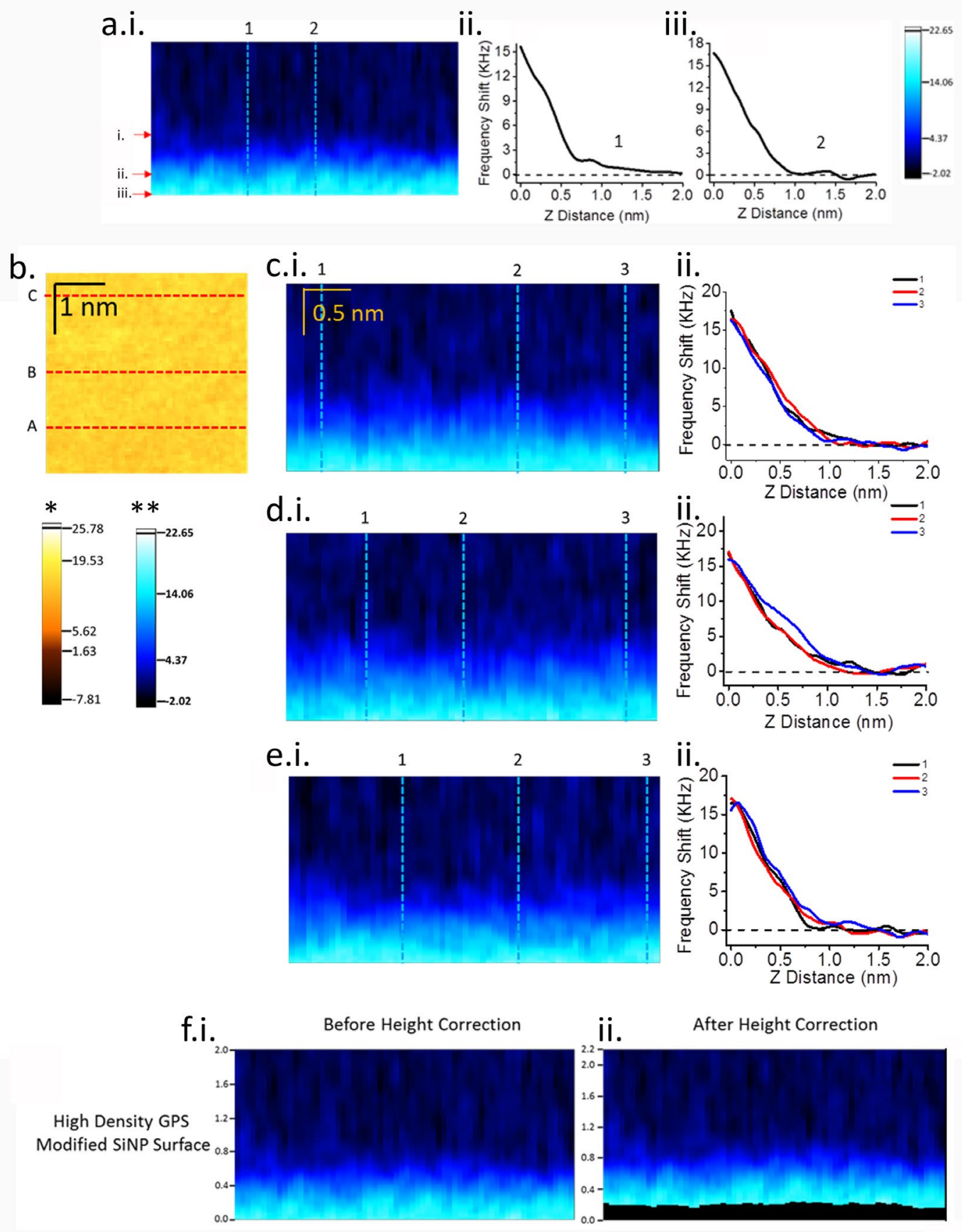

Figure 4. a. Representative 2D cross section at the GPS - SiNP interface (i), with representative 1D profiles taken at positions 1 (ii) and 2 (iii) on the SiNP surface. b. 2D topview image illustrating positions of cross sections and 1D profiles across a GPS - SiNP surface. Scanning area is $4 \mathrm{~nm} * 4 \mathrm{~nm} * 2 \mathrm{~nm}$. The $2 \mathrm{D}$ top view image is taken at $\mathrm{z}=0 \mathrm{~nm}$ and labelled with dotted lines (a-c) at the position of each 2D cross section (c.i., d.i., e.i.), which are also labelled with positions of 1D profiles taken at positions 1,2 and 3 that are labelled with black, red and blue lines in the 1D profiles, respectively (c.ii., d.ii., e.ii.). f. Before height correction (i) and after height correction (ii) 2D sections. Legend: * illustrates relative frequency difference for $b$. ** illustrates relative frequency difference for $c, d, e \& f$. The cantilever parameters: $A=1.65 \AA$, $f=1230 \mathrm{kHz}, \mathrm{Q}=10.3$ and $\mathrm{k}=65.25 \mathrm{~N} / \mathrm{m}$. The 3D $\Delta \mathrm{f}$ 
images parameters: The frequency and amplitude of the $z$ modulation during the 3D-SFM imaging were $195.3 \mathrm{~Hz}, 3 \mathrm{~nm}$, and $12.2 \mathrm{~nm} \mathrm{~s}-1$, respectively.
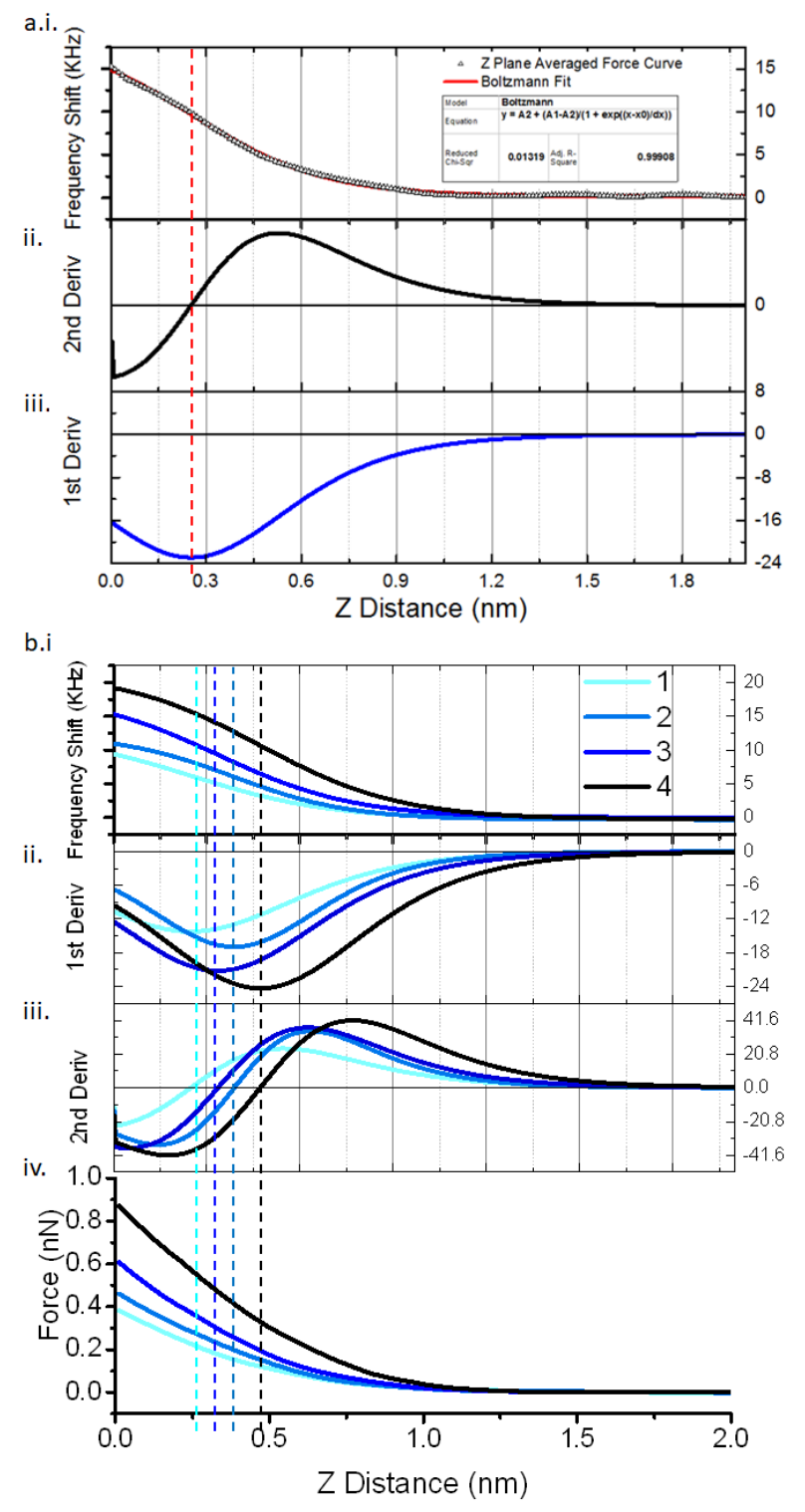

Figure 5. a. (i). Boltzmann Sigmoid fit with (ii) $1^{\text {st }}$ and (iii) $2^{\text {nd }}$ derivative of averaged $1 D$

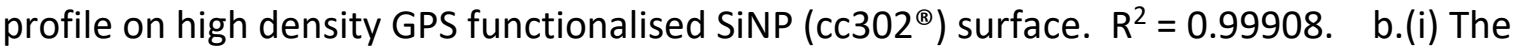
Boltzmann Sigmoid fitted averaged 1D frequency shift vs. Z distance curves of high density GPS modified SiNP with a function of frequency shift setpoints. The curves are labelled with number 1, 2, 3 and 4 which represent for setpoints at $9.45 \mathrm{KHz}, 11.08 \mathrm{KHz}, 15.62 \mathrm{KHz}$ and $19.58 \mathrm{KHz}$, respectively. Figure b. (ii and iii) represent for $1^{\text {st }}$ and $2^{\text {nd }}$ derivative of curves in figure b.(i). The force curves in figure b.(iv) are converted from experimental collected frequency shift vs $Z$ distance data based on Sader's method. The dash lines in figure 5.b indicate the transition point of constant compliance. 
a.

b.

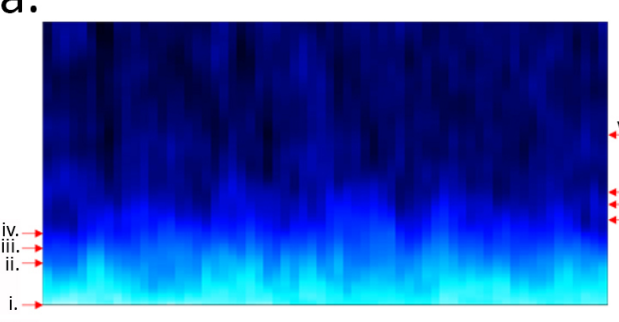

C.
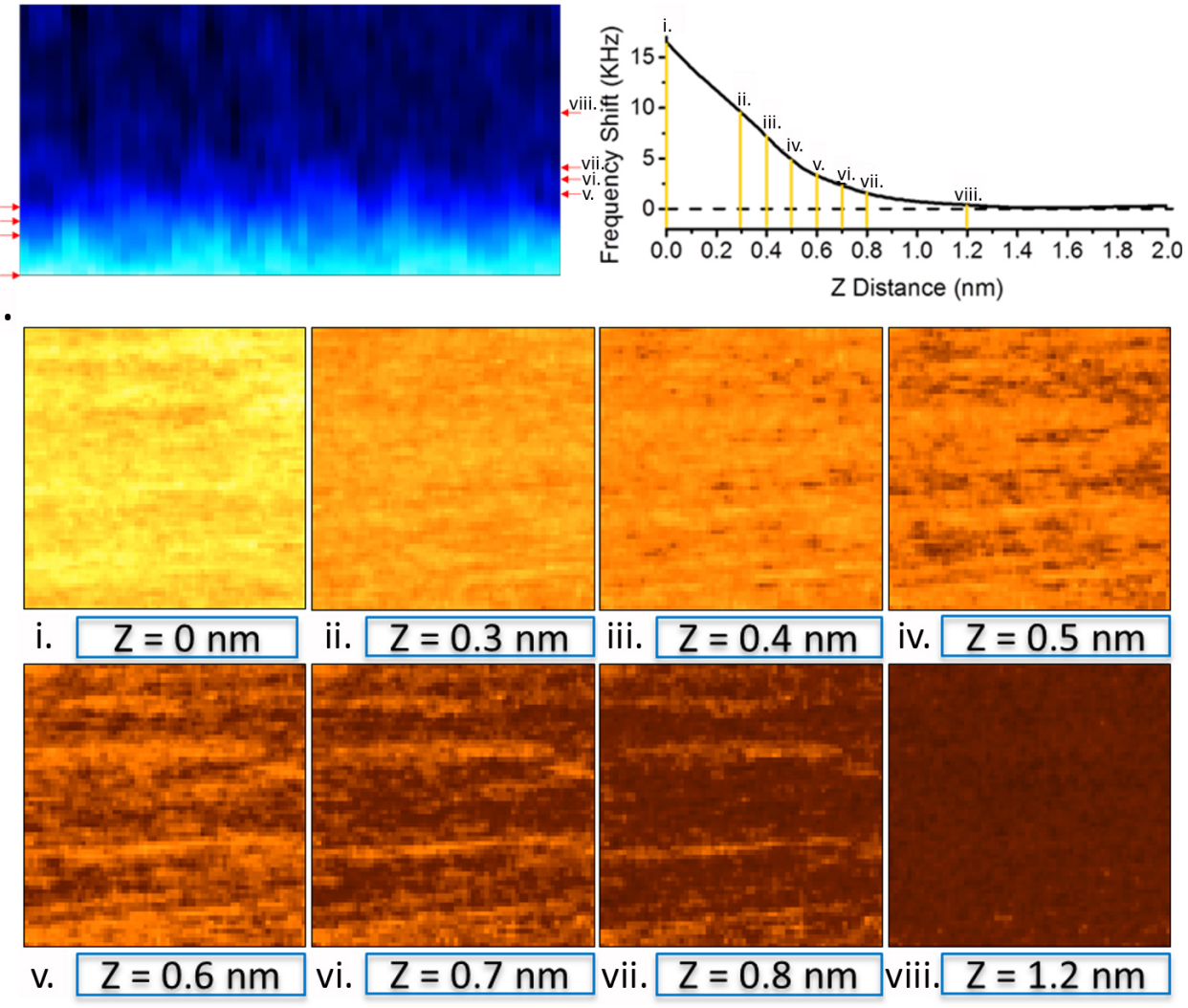

Figure 6. a. 2D cross section of GPS-SiNP surface. b. Averaged 1D profile from (a). c. Topview images at different $\mathrm{z}$ positions labelled from $\mathrm{i}$ - viii. correlating to $\mathrm{z}$-distances labelled in (a) and (b). 
a.
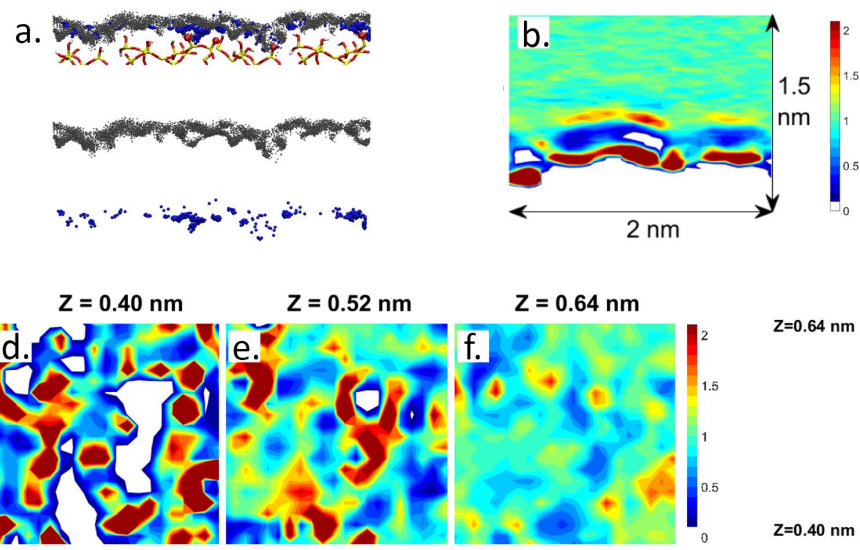
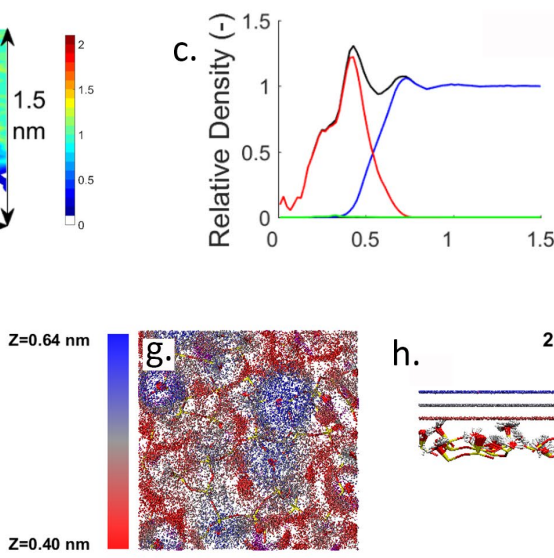

h.

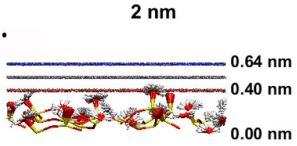

Figure 7. a. Density map of oxygen (grey) and sodium (blue) atoms on bare $\mathrm{SiO}_{2}$. b. Representative 2D cross-section of the density of water on silica. For (b) the colour scale refers to the relative atomic density of water oxygen atoms normalised by the average density of water more than $1.8 \mathrm{~nm}$ from the substrate. c. Averaged 1D density profiles vs Z distance curves for silica with total (black); interfacial water (red) and bulk-like water (blue). d-f. $X Y$ slices taken from a 3D relative atomic density map on bare $\mathrm{SiO}_{2}$ at different $\mathrm{Z}$ positions as indicated in (g). $Z$ height span of the contour plot is $0.02 \mathrm{~nm}$. The colour scale refers to the relative atomic density of water oxygen atoms normalised by the average density of water more than $1.8 \mathrm{~nm}$ from the substrate. g. Snapshot of water-oxygen atoms within $0.35 \mathrm{~nm}$ of the unmodified silica substrate from the $200 \mathrm{~ns}$ simulations, colorbar indicates the height above the underlying substrate. $h$. Schematic showing relative positions of (d-f). 


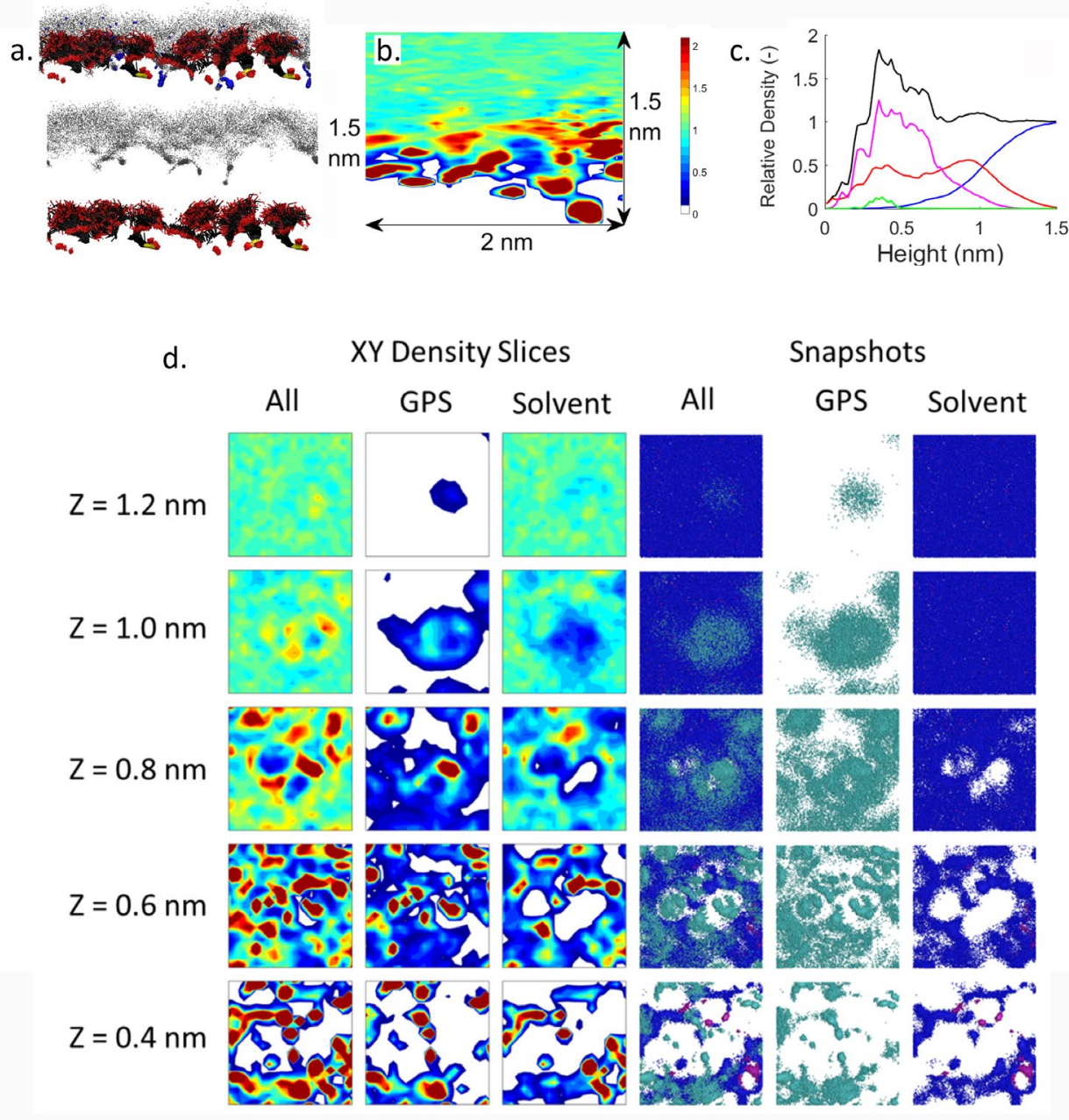

Figure 8. a. Density map of oxygen (grey) and sodium (blue) atoms on high-density GPS-

$\mathrm{SiO}_{2}$ surfaces. b. Representative 2D cross-sections of high density GPS modified silica surfaces. For (b) the colour scale refers to the combined relative atomic density of water oxygen atoms and all non-hydrogen atoms in the GPS ligand (C, $\mathrm{O}$, and the one $\mathrm{Si})$ normalised by the average density of water more than $1.8 \mathrm{~nm}$. c. Averaged 1D density profiles vs Z distance curves for high density GPS modified silica with total (black); interfacial water (red) bulk-like water (blue), GPS chain (magenta) and sodium (green). d. $2 \times 2 \mathrm{~nm}^{2} \mathrm{XY}$ contour slices of the relative atomic density at the high density GPS modified silica interface in $0.02 \mathrm{~nm} z$ distance bins ranging from 0.4 to $1.2 \mathrm{~nm}$ above the silica substrate). The colour scale refers to (All - left hand side) the combined relative atomic density of water oxygen 
atoms and all non-hydrogen atoms in the GPS ligand ( $\mathrm{C}, \mathrm{O}$, and the one $\mathrm{Si}),(\mathrm{GPS})$ relative atomic density of all non-hydrogen atoms in the GPS ligand only; (Solvent) relative atomic density of water oxygen atoms only, each normalised by the average density of water more than $1.8 \mathrm{~nm}$. 